\title{
Egzotik Hadronlar
}

\author{
Exotic Hadrons
}

\author{
Jale Y. SÜNGÜ̈ ${ }^{1}$ (D), Elşen VELi' ${ }^{1,2}$ (i) \\ ${ }^{1}$ Kocaeli Üniversitesi Fen-Edebiyat Fakültesi Fizik Bölümü, 41380 Umuttepe/İzmit \\ ${ }^{2}$ Kocaeli Üniversitesi Eğitim Fakültesi, 41380 Umuttepe/İzmit
}

Öz

Son yıllarda yapılan yüksek enerji fiziği deneylerinde Standart Model çerçevesinde bilinen parçacık yapıları dışında yeni parçacıklar dedekte edilmiştir. Bu gelişmeler, Standart Model ötesi fizik açısından oldukça önemlidir. İç yapısı nedeniyle egzotik parçacık diye adlandırılan bu parçacıkların hem deneysel, hemde teorik çalışmalara farklı bir yön vereceği düşünülmektedir. Bu parçacıklar bugüne kadar tam olarak açıklanamayan madde-antimadde asimetrisi, evrenin oluşumu, karanlık madde, karanlık enerji, nötron yıldızlarının iç yapısı gibi pekçok konu hakkında ipuçları verebilir. Ayrıca bu parçacıkların keşfi son yıllarda hadron spektroskopisine olan ilgiyi oldukça artırmıştır. Bu çalışmada, bugüne kadar tespit edilen egzotik parçacıkların kütle, kuantum sayıları, bozunma genişlikleri gibi temel özellikleri hakkında bilgi sunulacaktır.

Anahtar Kelimeler: Egzotik parçacık, gluontopu, hibritler, tetrakuark, pentakuark, hegzakuark, molekül yapılar, baryonyum

\begin{abstract}
In recent years, in the high energy physics experiments, new particles have been detected except for known particle structures in the Standard Model framework. These developments are also very important in terms of physics beyond the Standard Model. It is thought that particles in this different structure, which is called exotic particle due to internal structure, will give a different direction to both experimental and theoretical studies. These particles can give clues on the asymmetry of matterantimatter, the formation of the universe, the dark matter, the dark energy, the inner structure of neutron stars, and so forth. Moreover, the discovery of these particles has widely increased the interest in hadron spectroscopy in recent years. In this work, information on the basic properties of exotic particles, such as mass, quantum numbers, and decay widths, which are detected until now, will be presented.
\end{abstract}

Keywords: Exotic particle, glueball, hybrids, tetraquark, pentaquark, hexaquark, molecular structures, baryonium

\section{GİRIS}

Standart Modelin bir parçası olan ve 1964 yılında Gell-Mann ve Zweig tarafindan önerilen Kuark Modeli, hadronları en iyi açıklayan modeldir [1]. $\mathrm{Bu}$ modele göre, hadronlar kuark içeriklerine göre mezonlar $(q \bar{q})$ ve baryonlar ( $q q q$ veya $\bar{q} \bar{q} \bar{q})$ olarak sinıflandirılır. Standart Modele göre kuarklar arasındaki etkileşme gluonların alışverişi ile gerçekleşir. Kuark ve gluonlar arasındaki güçlü etkileşimi tanımlayan Kuantum Renk Dinamiği (KRD veya QCD), Abelyan olmayan $S U(3)$ simetrisine sahip yerel ayar alan teorisidir. KRD Lagranjiyeni aşağıdaki şekilde tanımlanır:

$$
L=\bar{\psi}_{i}\left(i \gamma^{\mu}\left(D_{\mu}\right)_{i j}-m \delta_{i j}\right) \psi_{j}-\frac{1}{4} G_{\mu \nu}^{a} G_{a}^{\mu \nu},
$$

burada

$$
\left(D_{\mu}\right)_{i j}=\partial_{\mu} \delta_{i j}-i g A_{\mu}^{a} T_{i j}^{a}
$$

kovaryant türev, $\psi_{i}$ kuark alanı ve $A_{\mu}^{a}$ gluon alanı, $\gamma^{\mu}$ Dirac matrisi ve $T^{a}=\lambda^{a} / 2, S U(3)$ ayar grubunun üreteçleridir. KRD lagranjiyeninden yararlanarak analitik olarak hadron spektrumunu elde etmek mümkün değildir. Büyük enerji bölgesinde veya küçük mesafelerde güçlü etkileşme sabiti küçük olduğundan, KRD'de seri açılımı kullanılarak hesap yapılabilir. Fakat düşük enerji

Sorumlu yazar/Corresponding Author: Jale YILMAZKAYA SÜNGÜ , Tel: 053269403 05, e-posta: jyilmazkaya@kocaeli.edu.tr Gönderilme/Submitted: 16.09.2017, Düzenleme/Revised:20.03.2018, Kabul/Accepted: 23.03.2018. 
bölgesinde veya uzak mesafelerde etkileşme sabiti büyük olduğundan, KRD lagranjiyenini kullanarak parçacıkların özelliklerini incelemek büyük zorluklar içermektedir. Bu enerji bölgesinde seri açılımı geçerli değildir ve parçacıkların incelenmesi pertürbatif olmayan yeni yöntemlerin kullanılmasını gerektirmektedir. KRD'de gluonların kendi kendileriyle de etkileşmeleri (özetkileşim) nedeniyle, bağlı gluon durumlarının da varolabileceği öngörülmektedir. Gluonların birbiri ile etkileşmesi teoriyi nonlineer yapmakta ve bazı özel durumlarda yaklaşık yöntemler kullanılarak teorik hesaplar yapılabilmektedir. KRD'ye göre renk kuantum sayısı nötr olacak şekilde bir parçacık oluşturmak üzere farklı kuark ve/veya gluon kombinasyonları da mümkündür [2].

Ayrıca derin elastik olmayan saçılmaların detaylı incelenmesi sonucu artık mezonların basit bir $q \bar{q}$ yapısına sahip olmadıklarını biliyoruz; Renkli kuarklar KRD vakumu ile etkileşme halinde olduğundan, kuark ve gluon yoğuşmaları kutuplanır ve mezonik dalga fonksiyonu, sanal $q \bar{q}$ çiftleri ve gluonik alan konfigürasyonlarını içerir. Bu nedenle Örgü (Lattice) KRD'den ve KRD'den ilham alan modellerden siradan spektrumun ötesinde öngörüler bekleniyor. $\mathrm{Bu}$ yeni durumlar bilinmeyen ve farklı üretim ve/veya bozunma özelliklerine sahip olabilir.

1974 yılında $J / \psi(c \bar{c})$ parçacı̆̆ının keşfinden sonra yaşanan en ilginç olaylardan birisi, 2003 yılında BaBar işbirliği ile $D_{s}(2317)$ parçacığının dedekte edilmesi olmuştur. Bu parçacığın keşfi ile yüksek enerji fiziğinde yeni bir dönem başlamıştır. Ardından CLEO, Belle, DO, CDF, LHCb, BES, v.b. işbirlikleri ile deneyler yapılmış ve çok sayıda yeni ve farklı yapıdaki parçacıklar bulunmuştur [38]. Standart olmayan doğalarından dolayı, bu yeni gözlemlenen parçacıklara "egzotik parçacıklar" adı verildi. $\mathrm{Bu}$ ilginç yapılar: $X Y Z$ parçacıkları, gluontopları (glueball) veya gluonyum, hibritler $(q \bar{q} g$ veya $q q q g)$, mezon molekülleri $(q q \bar{q} \bar{q})$, tetrakuarklar $(q \bar{q} q \bar{q})$, pentakuarklar $(q q q q \bar{q})$ ve

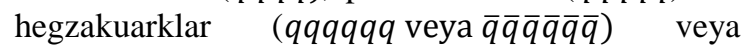
dibaryonlar $(q q q \bar{q} \bar{q} \bar{q})$. Son y1llarda geleneksel durumlarin listesine yeni bulunan, $h_{c}, \chi_{c 2}(2 P), B_{c}^{+}$ve $\eta_{b}(1 S)$ durumlarının eklendiği çalışmalar oldu. Bunlara ilaveten beklenmedik ve hala net bilgilere sahip olamadığımız $X(3872)$ ve bir düzineden fazla diğer çarmonyum ve botonyum benzeri $X Y Z$ durumları keşfedildi. Bu parçacıkların Standart Kuark Modelinin dişında kaldığı düşünülmektedir. Bunların birçoğunun hala deneysel doğrulanmaya ihtiyacı vardır $[9,10]$.

$\mathrm{Bu}$ makalede son yıllarda keşfedilen çok sayıda egzotik parçacık sınıflarından ve bu yeni tipteki parçacıkların temel özelliklerinden kısaca bahsedilecektir. Bu yeni durumların daha hassas ve yeni ölçümler yapılarak doğrulanmas1 gerekmektedir.

\section{EGZOTIK PARÇACIKLARIN SINIFLANDIRILMASI}

Son yıllarda yüksek enerji fiziği laboratuarlarında bildiğimiz parçacık yapılarından farklı çok sayıda egzotik mezon ve baryon tespit edilmesi bu konudaki deneysel ve teorik çalışmalara hız kazandırmıştır. Egzotik parçacık terimi, Standart Modeldeki herhangi bir parçacığın özelliklerine benzemeyen farklı özelliklere sahip parçacıkları tanımlamakta kullanılır.

Bu çalışmada, kuark ve gluon içerikli olup Standart Kuark Model ile açıklanamayn egzotik parçacıklar ele alınacaktır. Literatürdeki bazı çalışmalarda Büyük Birleşik Teorilerinin X\&Y ayar bozonları, Higgs bozonu, monopol (tek manyetik kutup) ve benzeri egzotik parçacıklar olarak yeralmaktadır. Deney teknolojisindeki son gelişmeler, aranan parçacığa ait sinyaller ile arkaalan sinyallerinin daha iyi ayırt edilmesi sonucunda egzotik parçacıkların dedekte edilmesini kolaylaştırmıştır. $\mathrm{Bu}$ parçacıklar genel olarak aşağıdaki şekilde sinıflandırılabilir:

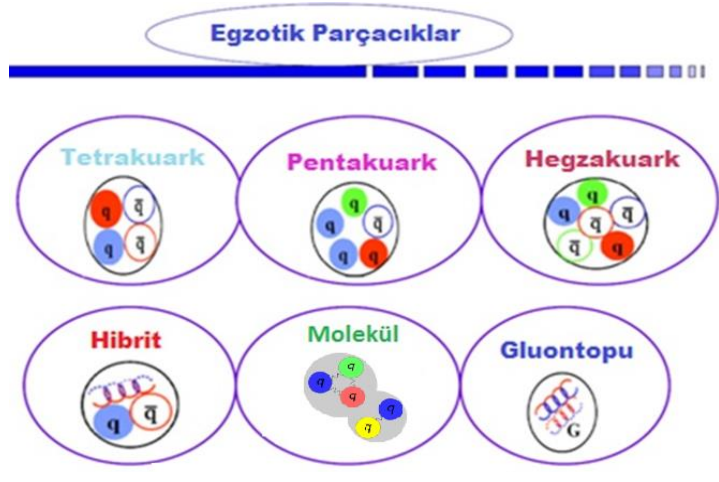

Şekil 1. Egzotik parçacık sınıfları [11].

Gözlemlenen hadronik spektrumun açıklanmasında, özellikle de ağır çeşni sektöründe Standart Kuark Modeli oldukça başarılıdır. Bununla birlikte, hafif hadronik spektrumun açıklanmasında Kuark Model yetersizliklere sahiptir. Böylece hafif hadron spektroskopisini inceleyerek KRD vakumu, ebedi hapsolma ve benzeri özellikler anlaşılabilir.

\subsection{Gluontopları}

İlk kez Fritzch ve Gell-Mann, 1972'de gluonlardan oluşan mezonlar olabileceği fikrini önesürdüler [12]. Bu, protonların kuark yapısının keşfedildiği 1968 yılındaki SLAC derin-elastik olmayan saçılma deneyinden yaklaşık dört yıl sonra idi. 1975'de Fritzsch ve Minkowski [13] gluontopu durumlarının yeni bir madde türü olabileceğini, bu durumların varlığının doğrudan kuark-gluon alan teorisinin sonucu olduğunu söylediler. Literatürde 
gluonyum terimi bir $g \bar{g}$ durumu için kullanılırken gluontopu kavramı ise daha genel anlamda kullanılmaktadır.

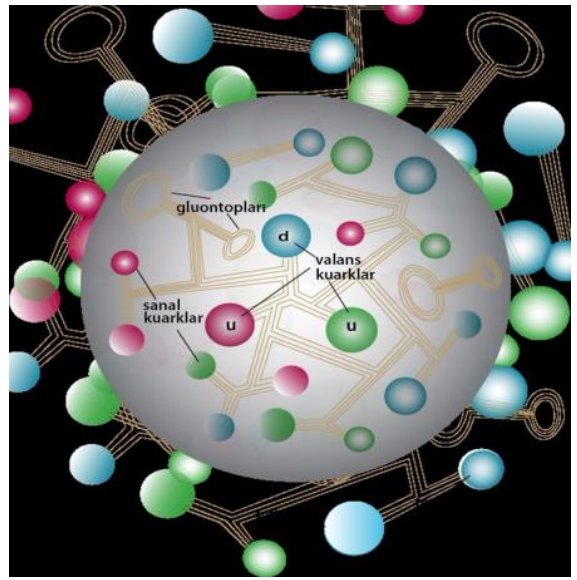

Şekil 2. Protonun modern tasviri, $+1 e$ elektrik yükü üreten üç valans kuarktan çok daha fazlasını içerir. $\mathrm{Bu}$ üç kuark, protonun kütlesinin yalnızca yüzde 2'sini oluşturur. Gerisi sanal kuarkların ve yapışkan topların "denizinden" gelir [14].

1977'de D. Robson [15] makalesinde skaler mezonların ideal dokuzlu karışım olabileceğini belirtti. Ayrica kuantum sayıs1 $J^{P C}=0^{-+}$ olan $\eta^{\prime}$ (958)'nin büyük bir kısmının gluontopu olarak ele alınabileceğini öngördü. $\mathrm{O}$ zamandan bu yana yaklaşık 40 yıl geçmesine rağmen hala net bir bilgiye sahip değiliz.

Skaler mezonları anlamak özellikle önemlidir, çünkü bu parçacıklar vakumla aynı kuantum sayılarına sahiptirler $\left(J^{P C}=0^{++}\right)$. Bu nedenle vakum içine yoğunlaşabilir (yani vakum bu parçacıkları sogurup veya yayabilir) ve $U\left(N_{f}\right) \times$ $U\left(N_{f}\right)$ bir global kiral simetriyi kirabilirler. Bu simetrinin nasıl kırıldığının ayrıntıları parçacık fiziğinin önemli problemlerinden biridir. Skaler mezonların iç yapıları uzun süredir devam eden bir bulmacadır. Yaklaşık son 40 yıldır fizikçiler gluontopu denilen ve sadece gluonlardan oluşan hipotetik parçacıkları aramaktadır. Bu parçacıklar kararsız olduğundan sadece bozunmaları analiz edilerek dolaylı yollardan gözlemlenebilirler.

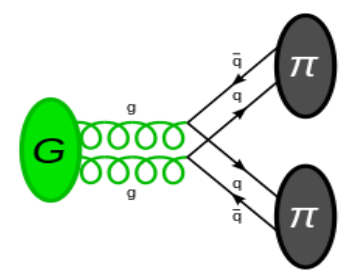

Şekil 3. $\pi$ mezonlara bozunan gluontopları [16].

Skaler gluontopu spektrumunu incelemek için çok sayıda deney yapılmış ve özellikle 1 GeV'in üstündeki bölge önem kazanmıştır. Hafif pseudoskaler mezonların aksine skaler mezonların özelliklerini anlamak çok daha önemlidir ve düşük enerjideki KRD için hala netleştirilmesi gereken bir konudur. Kütlesi $1 \mathrm{GeV}^{\prime}$ in altındaki durumlar: $f_{0}(500)$ (veya sigma $(\sigma)$ mezon) ve $f_{0}(980)$, $K_{0}^{*}(800) \quad$ (veya kappa $(\kappa)$ mezon) ve $a_{0}$ (980) mezonlar basit $q \bar{q}$ gösterimi ile açıklanamaz. Bununla birlikte kütlesi $1 \mathrm{GeV}$ 'in üstündeki $f_{0}(1370), \quad f_{0}(1500)$ ve $f_{0}(1710)$, $K_{0}^{*}(1430)$ ve $a_{0}(1450)$ gluontoplarına aday parçacıklar olarak görülebilir. $a_{0}(980)$ ve $f_{0}(980)$ parçacıkları literatürde geniş bir şekilde tartışılmakta ve bunların gluontopu olabileceği gibi $K \bar{K}$ molekül durumu, tetrakuark durumu veya $q \bar{q}$ mezon durumları olabileceği de düşünülmektedir. Ayrıca $f_{0}(1710)$ adı verilen parçacığın uzun zamandır aranan gluontopu olduğuna dair güçlü deliller önesürülmüştür. Basitleştirilmiş model hesaplamalar1 $f_{0}(1500)$ ve $f_{0}(1710)$ 'un gluontopu adayları olduğunu göstermektedir [17].

Örgü Teorisi, KRD toplam kuralları, akı tüpü ve gluon bileşenli modeller en hafif gluontoplarının kuantum sayılarının $J^{P C}=0^{++}$ve $J^{P C}=2^{++}$ olduğu konusunda hemfikirdir. Örgü Teorisine göre, gluontoplarının kütleleri uyarılmış durumlar $\left(2^{++}\right)$için yaklaşık $2300 \mathrm{MeV}, 0^{++}$kuantum numaralı temel durumlar için ise $1600-$ $1700 \mathrm{MeV}$ kütle aralığındadır [18-21]. $0^{+-}$ve $0^{--}, 0^{+-}, 1^{-+}, 2^{+-}$, vb. gluontopu durumlarının kütlesinin $2 \mathrm{GeV}$ üzerinde olması beklenmektedir [21]. Ayrıca Örgü Teorisine göre gluontopları kompakt nesnelerdir ve skaler gluontoplarının yarıçapı yaklaşık 0.3 fm'dir [22].

Gluontopu üretimi için en göze çarpan süreç 1şınsal $J / \psi$ bozunumudur. $J / \psi$ 'nin bozunma genişliği dardır; $D \bar{D}$ eşiği $J / \psi$ kütlesinin üstündedir ve OZI kuralı, $c \bar{c}$ sisteminin hafif kuarklara dönüşmesini engeller. Bozunmaların çoğunda, $J / \psi$, üç gluona bozunarak hadronlara dönüşür. Ancak $J / \psi$ iki gluon ve bir fotona da bozunabilir. Foton dedekte edilebilir, iki gluon ise etkileşime gluontopları oluşturabilirler. Ayrıca, yapılan çalışmalar, merkezi çarpışmalarda gluontoplarının bol miktarda üretileceğini gösterir. Merkezi çarpışmalarda iki hadron birbirine hemen hemen dokunmadan geçer ve ileri yönde kırınarak dağılırlar, valans kuarklar ise değiş-tokuş yapmaz. Süreç genellikle PomeronPomeron saçılması olarak adlandırılır. Üretim sürecine valans kuarkların katılmaması merkezi çarpışmaları gluontoplarının araştırılması için uygun bir çalışma alanı yapar. Ayrıca $p \bar{p}$ yokoluşunda, kuark-antikuark çiftleri gluontopları oluşturabilir.

2014 yilinda Thomas Jefferson Ulusal Hızlandırıcısında (JLAB) başlayan GlueX deneyi gluontoplarını dedekte edebilmek amaciyla dizayn edilmiştir. Bu deneyin ana amacı kuarkları birarada 
tutan gluon alanının uyarılmasıyla oluşan egzotik mezonların spektrum haritasını çıkarıp, KRD' nin hala tam olarak açıklanamayan ebedi hapsolma olayının doğasını anlamaktır. Deneyde egzotik durumları oluşturmak için fotoüretim (bir nükleon üzerinden gerçek bir fotonun saçılması) tekniği kullanılmaktadır. GlueX dedektöründe $12 \mathrm{GeV}$ enerjilere kadar çıkıldı. Gluontopu spektroskopisinin en önemli sorunu, gluontoplarının bilinen ve egzotik hadron durumları ile karışması ve bu nedenle deneylerde tespit edilmesinin zor olmasidir. Gelecekte GlueX, CLAS12, BESIII ve PANDA deneylerinden elde edilecek verilerle gluontopları hakkında daha net bilgilere ulaşılması beklenmektedir.

\subsection{Tetrakuarklar}

1977 y1lında Jaffe $\kappa, a_{0}(980), f_{0}(980)$ ve $\sigma$ parçacıklarının bilinen parçacık yapısında olmadığını öngören ilk modeli önesürdü [23]. Bu parçacıklar tetrakuark yapıda olabilirlerdi. Egzotik parçacıklardan biri olan tetrakuarklar, belirli bir renk kuantum sayısına sahip iki kuark ve uygun iki anti-renk yüküne sahip, iki antikuarkın biraraya gelmesi ile $([q \bar{q}][q \bar{q}])$ oluşurlar. $\mathrm{Bu}$ parçacıkların keşfi, yıldızların evrimini anlamak bakımından oldukça önemlidir. Bilim adamlarına göre nötron yıldızlarındaki maddenin bir kısmı nötronlar, bir kısmı ise standart kuark modeli çerçevesindeki hadronlar, bir kısmı ise egzotik parçacıklar olabilir. Tetrakuarklar, $B$ mezonların bozunumu sonucu oluşurlar ve yaşam süreleri yaklaşık $10^{-24}$ saniyedir. Bu yeni parçacıklar farklı uyarılmış durumlarda bulunarak, farklı kütleler kazanırlar.

2003 yılında Japonya'daki Belle deneyinde ilk kez dört kuark içerdiği düşünülen X(3872) parçacığının gözlemlendiği bildirildi [4]. Ancak yeterli kanıt mevcut değildi. 2007 yılında keşfedilen $Y(4660)$ parçacığının da bir tetrakuark olabileceği önesürüldü $\quad[25,26]$. 2009'da Fermilab $Y(4140)$ adını verdikleri yeni bir parçacık bulduklarını duyurdular [27]. Bu parçacık iki mezona bozunuyordu. Bu parçacığın tılsımlı ve anti-tılsımlı kuark içerebileceği düşünüldü. Daha sonra 2010 yılında $Y(5 S)$ mezonunun tetrakuark rezonansı olduğu önesürüldü [28]. 2008 yılında ilk kez Belle İşbirliği, $B$ mezonların bozunumunda $Z$ (4430) adı verilen bir egzotik parçacığ 1 dedekte ettiklerini bildirdi [29]. Bu parçacık daha sonra $\mathrm{LHCb}$ tarafindan proton-proton çarpışmasındaki 25.000 'den fazla veri analiz edilerek $B$ mezon bozunumu ile doğrulandı [30].

2013'de iki bağımsız grup $Z_{c}$ (3900) adını verdikleri parçacığı keşfettiklerini bildirdiler [31]. $\mathrm{Bu}$ parçacık $Y(4260)$ parçacı̆̆ 1 bozunduğunda gözlenmiştir ve dört kuark içerdiği düşünülmektedir. 2015 yılında, Çin'deki BESIII (Beijing Elektron Pozitron Çarpıştırıcısı III) işbirliği nötr $Z_{c}(3900)$ parçacı̆̆ını dedekte etti. Bu parçacık bir nötr pion ve bir mezona bozunuyordu. $\mathrm{Bu}$ sonuçlar $B^{+}$mezonunun $J / \psi, \phi$ ve $K^{+}$ mezonlarına bozunması sırasında gözlemlendi [32].

Sonuç olarak, 2014 yılında LHC tetrakuarkların varlığını doğruladı ve bu parçacıkların atomun yapısına benzer şekilde yapıya sahip olduğunu bildirdi. Bu yapılarda kuarklar daha yüksek enerji seviyelerine atlayıp, uyarılabiliyordu. Kuarkların enerji konfigürasyonu, egzotik yapıların kütle ve kuantum sayılarını belirlemekteydi. Daha büyük kütlelere sahip olan gözlemlenen yeni parçacıklar ise, $\quad X(4140), X(4274), X(4500)$ ve $X(4700)$ diye adlandırıldı [33]. Her dört parçacıkta aynı kuark yapısına sahip olmasına rağmen, her biri farklı kütle ve kuantum sayılarına sahiptir. $\mathrm{Bu}$ parçacıkların herbiri iki $c$ ve iki $s$ kuark içerir.

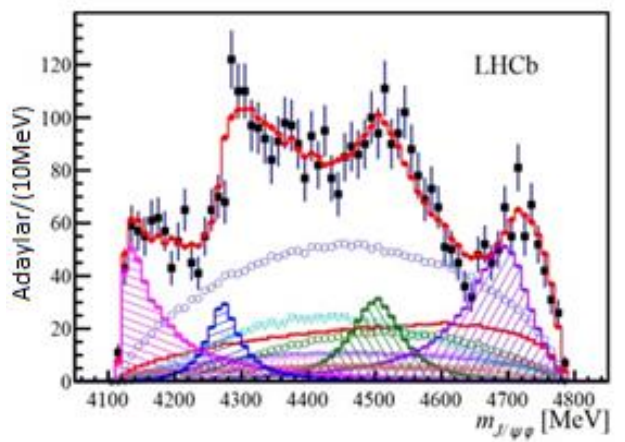

Şekil 4. Grafiğin alt kismındaki dört pik $X(4140), X(4274), X(4500)$ ve $X(4700)$ egzotik parçacıklarını işaret eder (Görüntü: CERN, [33]).

Temmuz 2015'de Fermilab Tevatron çarpıştırıcısında gerçekleştirilen DO deneyi sonucunda $5.1 \sigma$ istatistik ağırlık ile $X(5568)$ adı verilen yeni bir parçacık keşfedildiği duyuruldu [34]. Aslında Tevatronun çalışması 2011 yılında durdurulmuştu. Ama çarpışma sonucu elde edilen milyarlarca verinin analizi ancak yapılabilmişti. Parçacık fiziğinde $5 \sigma$ bir keşif yapmak için gerekli olan istatistik ağırlıktan daha büyüktür. $\mathrm{Bu}$ parçacı̆̆ın bir $b, s, u$ ve $d$ kuarklarını içerdiğini öngördüler. Bu parçacığın en ilginç özelliği, diğer tetrakuark ve pentakuarklara benzememesidir; dört farklı çeşnili kuark ve antikuark içerir. Ancak bu keşif CERN tarafından henüz doğrulanmamıştır. Ancak bu konuda LHCb'de çalışmalara devam edilmektedir [35].

\subsection{Molekül Yapılar}

Kuark modelde, dörtlü-kuark durumları genellikle iç yapılarına göre $[q q][\bar{q} \bar{q}]$ ve $[q \bar{q}][q \bar{q}]$ olarak sinıflandırılır. $\mathrm{Bu}$ iki tip dörtlü kuarklardan ilki 
$[q q][\bar{q} \bar{q}]$ tetrakuark durumu olarak adlandırılmış ve MIT torba modeline göre incelenmiştir. Hafif kuark içeren bu tür egzotik parçacıklara hafif tetrakuarklar denir. İkinci tipi ise iki hafif mezonun birbiri ile etkileşmesi sonucu oluşur ve molekül olarak adlandırılır. Bu iki yapının iç dinamiklerinin birbirinden farklı olması beklenir. $\mathrm{Bu}$ farklılık kendini, bu parçacıkların güçlü bozunmalarıyla sergileyebilir. Hadronik bir molekül, güçlü kuvvet ile birbirine zayıf bağlı mezon-mezon, baryonbaryon veya mezon-baryon kümesidir denilebilir.

Beklenmedik bir şekilde $X Y Z$ olarak bilinen çok sayıda çarmonyum benzeri durumların keşfi, hadronik spektrumun teorik olarak daha detaylı incelenmesini gündeme getirmiştir. Tılsımlı hadron sektöründe de ( $D$ ve $D_{s}$ mezonlar) benzer bir şaşkınlık yaşandı. $B$ fabrikaları çok dar bozunma genişliğine sahip iki parçacık tespit etti: $D_{s 0}^{*}(2317)^{ \pm}$ve $D_{s 1}(2460)^{ \pm}[3]$. Bu durumlar için ölçülen deneysel kütle ve genişlikler Standart Kuark Modellerinden beklenenden çok daha küçük ve dardı. Bu parçacıkların kütlesi $c \bar{s}$ mezonlar için beklenen kütleden daha küçük bir değere sahiptir. $\mathrm{Bu}$ nedenle $D_{s 0}^{*}(2317)^{ \pm}$ve $D_{s 1}(2460)^{ \pm}$dörtlü tetrakuark durumları veya $D K\left(D K^{*}\right)$ molekül durumu olarak yorumlanabilirler [36,37].

2003'te Belle deneyi tarafindan keşfedilen $X$ (3872) ve 2007 'de keşfedilen $Z$ (4430), molekül grubundaki en iyi adaylardır. Ayrica $Z_{b}(10610)$ ve $Z_{b}(10650)$ durumları da molekül yap1 adayıdırlar. $X(3872)$ 'nin $D \bar{D}^{* 0}$ eşik kütlesine ve bozunma özelliklerine yakın olması onun $J^{P}=$ $1^{++}$kuantum sayılı zayıf bağlı bir durum olarak kabul edilmesine sebep oldu. $D \bar{D}$ kütle eşiği üzerinde dedekte edilen durum sayısı potansiyel modelin öngördüğü çarmonyum durumlarından çok daha fazladır. Bu tür ağır kuark içeren tetrakuark durumlarına çarmonyum benzeri veya "XYZ parçacıkları" denir. Bu durumlar arasında kuantum sayıs1 $J^{P}=1^{--}$olan pekçok vektör parçacık vardir: $Y$ (4260), $Y(4360)$ ve $Y(4660)$ [38].

2017 yılının Mart ayında Beijing Spektrometre Deneyi (BESIII), yeni çarmonyum benzeri durumların gözlendiğini bildirdi. Deney, BEPCII hizlandırıcisında BESIII dedektörü ile $e^{+} e^{-} \rightarrow$ $\pi^{+} \pi^{-} J / \psi$ ve $e^{+} e^{-} \rightarrow \pi^{+} \pi^{-} h_{c} \quad$ tesir kesitlerini yüksek hassasiyetle ölçmüş ve her iki bozunma modunda da rezonans yapılar gözlenmiştir. İlk yap1 $Y(4220)$ yaklaşık $4.22 \mathrm{GeV}$ kütlesi ile her iki modda da görüldü. $Y(4320)$ parçacı̆̆ $1 \quad e^{+} e^{-} \rightarrow \pi^{+} \pi^{-} J / \psi$ sürecinde, $Y(4390)$ ise $e^{+} e^{-} \rightarrow \pi^{+} \pi^{-} h_{c}$ reaksiyonunda görüldü [39]. Bunlar vektör parçacıklardır ve bilinen vektör çarmonyum ve çarmonyum benzeri herhangi bir durumdan farklıdırlar. Yapılan deneylerde kütleleri 4 ile $4.6 \mathrm{GeV}$ aralığında bulunan çok sayıda vektör parçacıklar gözlemlenmiştir. Bu bölge, bir tılsımlı kuark-antikuark içeren birçok egzotik hadron için kütle bölgesidir. $\mathrm{Bu}$ durumların araştırılması molekül yapıların anlaşılması bakımından oldukça önemlidir.

\subsection{Baryonyum, Dibaryon ve Hegzakuarklar}

Altı kuarktan, farklı yapıda parçacıklar oluşabilir. Eğer altı kuarktan oluşan bir parçacık varsa, baryon sayısı ikidir ve bu parçacığa "dibaryon" adı verilir. Üç kuark ve üç anti-kuarktan oluşan bir durum için baryon sayısı sıfira eşittir ve bu duruma "baryonyum" denir. Deneylerde baryonyum durumları, mezonlar ve tetrakuarklar ile karışabilir. Tam tersine, dibaryonlar, baryon say1s1 korunumundan ötürü sıradan hadronlarla karışmaz ve daha kolay tespit edilebilirler. Ayrıca üç kuark ve üç antikuarktan oluşan sistemlere "hegzakuark" da denir. Tetrakuarklar ve pentakuarklar gibi, hegzakuarklar da çoklu kuark ya da bir baryonbaryon molekülü şeklinde varolabilirler.

İki nükleonun bağlı durumu olarak öngörülen baryonyumun keşfi tam olarak netlik kazanmamıştır. Bugüne kadar sadece birkaç olası baryonyum aday1 belirlendi. Sadece $p \bar{p}$ yokoluşunda görülen $[40,41] f_{2}(1565)$ durumunun baryonyum adayı olduğu düşünülmektedir. Belle deneyinde $\sqrt{s}=4.63 \mathrm{GeV}$ civarında $e^{+} e^{-} \rightarrow$ $\Lambda_{c}^{+} \Lambda_{c}^{-}$bozunmasinda gözlenen sinyal $Y(4660)$ tılsımlı baryonyum olarak yorumlanmış ve tam olarak netlik kazanmamıştır $[42,43,44]$. Şimdiye kadar gözlemlenen tek altı kuark içerikli parçacık, $u$ ve $d$ kuarklardan oluşmuş $d^{*}(2380)$ dibaryondur. Hegzakuarkların yaşamının 2/3'ünü kompakt bir hegzakuark formunda, 1/3'ünü ise bir molekül olarak geçirdiği düşünülüyor. Edinburgh nükleer fizik grubu, d*(2380)'in gerçek doğasını saptamak ve daha ağır kuark sektörlerinde diğer durumların varlığını ortaya koymak için bir dizi yeni deneysel çalışmaya öncülük etmektedir [45].

\subsection{Hibrit mezonlar}

Standart kuark modelinde mezonlar $q \bar{q}$ yapısına sahip olup, $I\left(J^{P C}\right)$ kuantum sayıları ile karakterize edilirler. Burada $I$ izospin, $J$ toplam açısal momentum, $P$ parite ve $C$ yük eşleniğidir. $L$ kuark ve antikuarkın göreli yörüngesel açısal momentumu, $S$ ise $q \bar{q}$ çiftinin toplam iç açısal momentumu olmak üzere, $P=(-1)^{L+1}$ ve $C=(-1)^{L+S}$ ile verilir ve mezonların kuantum sayılar1 $J^{P C}=0^{-+}, 0^{++}, 1^{--}, 1^{+-}, 1^{++}, 2^{--}, 2^{-+}$, $2^{++}, .$. şeklindedir. Ancak KRD teorisine göre, mezon spektrumu oldukça zengindir. Uyarılmış gluon alanları mezonun kuantum sayılarına katkı verir. Bu tip parçacıklar yani, bir $q \bar{q}$ ve salınım yapan gluon sistemi hibrit olarak adlandırılır. Gluon alanları vakum durumundan daha farklı kuantum sayıları taşıyabileceklerinden, hibritler $J^{P C}=$ $0^{--}, 0^{+-}, 1^{-+}, 2^{+-}, \ldots$ egzotik kuantum sayılarına 
sahip olabilirler. Yüksek hassasiyetli yeni deneyler doğadaki bu tip yapıların varlığı hakkında ipuçları vermektedir.

Hibritlerin kütlelerinin yaklaşık $2 \mathrm{GeV}$ civarında olması ve iki mezona bozunması beklenmektedir. Kuantum sayıları $0^{-+}, 1^{-+}, 1^{--}$ve $2^{-+}$olan temel durumdaki hibrit parçacıkların kütle değerlerinin $1.7-1.9 \mathrm{GeV}$ aralığında olması bekleniyor. Örgü Teorisine göre ise $1^{-+}$egzotik kuantum sayılarına sahip hibritlerin kütlesi yaklaşık $1.9 \pm$ $0.2 \mathrm{GeV}$ 'dir[46,47]. Araştırmacılara göre $\pi_{1}(1370)$ en düşük kütleli egzotik mezondur. Ancak bu parçacık hibrit olabileceği gibi, tetrakuark da olabilir. Kuantum sayısı bize bu parçacığın iki olasıllktan hangisi olduğu konusunda bir ipucu vermez. Ayrica $\pi_{1}(1440), \pi_{1}(1625)$ ve $\pi_{1}$ (2000)'inde hibrit olma ihtimali görülüyor $[48,49]$. Bir diğer $1^{-+}$durumu olan $\pi_{1}(1600)$ 'ün COMPASS deneyinde $190 \mathrm{GeV}$ enerjili pionların kurşun bir hedefe çarptırılarak $\rho \pi$ 'ye bozunduğu dedekte edildi [50]. Ayrica $J^{P C}=0^{-+}, 1^{--}$ve $2^{-+}$olan hibrit adaylarının bulunduğu da rapor edildi [51,52].

\subsection{Pentakuarklar}

Yüksek enerji fizikçileri dört kuark ve bir antikuarktan oluşan baryonların varolabileceğini önesürdü. 1997'deki bir makalesinde Diakonov ve grubu $u, d$ ve $s$ kuarklardan oluşan, spini $1 / 2$ ve pozitif pariteli bir egzotik baryonun varolabileceğini öngördü [53]. Bu gibi parçacıklar renk kuantum sayısı açısından nötrdür, kararsız olurlar ve kolaylıkla baryonlara ve mezonlara dönüşürler.

1986'da Moskova ITEP'deki kabarc1k odas1 deneylerinde 1.54GeV'lik bir pik gözlendi. 1997 y1lında Petersburg Nükleer Fizik Enstitüsünden bir grup bilim adamı pentakuarkların varlığını önesürdü. 1997/98 yılında Almanya Bonn'daki ELSA hizlandirıcisında alınan verilerin $25 \mathrm{MeV}$ 'den küçük bozunma genişlikli ve $1.54 \mathrm{GeV}$ kütleli bir parçacı̆̆ın varlığına işaret ettiği bildirildi. 2002'de Osaka üniversitesi'nden Prof. Nakano'nun grubu bir karbon hedefi X-ışınları ile bombardıman ederek pentakuarkların varlığına dair ipuçları buldular [54]. Pentakuarkların varlığına dair son kanıt 2003 yılında Amerika'daki Jefferson laboratuarından geldi. Deneyde bir döteryum hedefi fotonlarla bombardıman edildi. $22 \mathrm{MeV}$ bozunma ömrüne sahip, $1.54 \mathrm{GeV}$ kütleli beș kuarktan oluşan bir baryona ait deliller bulundu [7]. uudd $\bar{s}$ içeriğine sahip bu yeni parçacığa Theta-plus $\left(\Theta^{+}\right)$adı verildi. Parçacığın toplam elektrik yükü
$+1 e$ idi ve yaklaşık $10^{-20} s^{\prime}$ de bir $K^{+}$mezon ve bir nötrona dönüşüyordu.

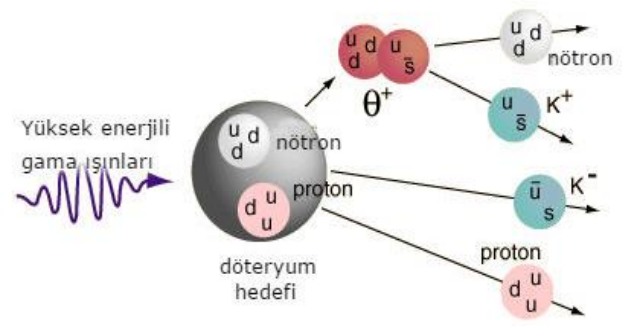

Şekil 5. Bir döteryum hedefinin fotonlarla bombardıman edilmesi ve pentakuark parçacığının oluşumu [55].

Pentakuarkları destekleyen daha sonraki kanıtlar 2e elektrik yüklü, $1862 \mathrm{MeV}$ kütleli, $s s d d \bar{u}$ ve

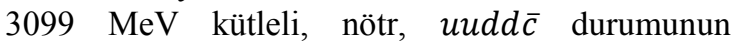
gözlenmesi ile geldi. Bu parçacıklara sırasıyla $\Phi(1860)$ and $\Theta_{c}(3100)^{0}$ adları verildi [56,57]. 2015 yilında LHC'de yapılan deneyler sonucu proton kütlesinden yaklaşık 4.7 kat ve diğeri bundan biraz daha ağır iki bilinmeyen parçacık tespit edildi. Yapılan analizlere göre bu parçacıklar bir $c$, bir $\bar{c}$, iki $u$ ve bir $d$ kuark içermekteydi. Gözlenen egzotik baryonlar 2015 yılında LHCb işbirliği ile $\Lambda_{b}^{0} \rightarrow J / \psi p K^{-}$bozunması ile keşfedilen ve iki tılsımlı kuark içeren pentakuarklar $P_{c}^{+}(4380)$ ve $P_{c}^{+}(4450)$ 'dir [7]. $\mathrm{Bu}$ tür parçacıkların geleneksel Standart Kuark Model ve onun yeni versiyonları ile açıklanması mümkün olmamış ve bu parçacıkların özelliklerini açıklamak için Tek-bozon-değiştokuş modeli (OBE), Tekpion-değiştokuş modeli (OPE) gibi çeşitli teorik modeller önesürülmüştür.

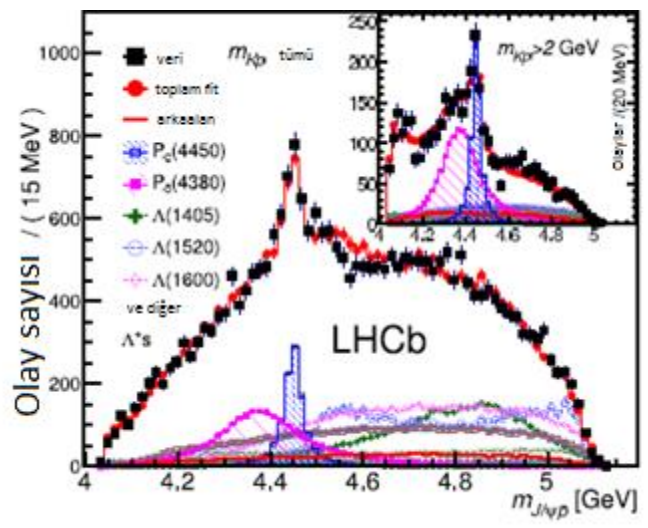

Şekil 6. LHCb işbirliği ile keşfedilen $P_{c}^{+}(4380)$ ve $P_{c}^{+}(4450)$ baryonlar (Görüntü: LHCb [7]). 
Tablo I. Egzotik mezon ve baryon adayları ( $q=u, d, s$ kuark).

\begin{tabular}{|c|c|c|c|c|c|c|}
\hline Parçacık & $\begin{array}{l}\text { Olası } \\
\text { İçerik }\end{array}$ & $\mathbf{J}^{\mathrm{PC}}$ & $\Gamma(\mathrm{MeV})$ & Yorum & Deney & $\begin{array}{l}\text { Gözlemlendiği } \\
\text { Bozunum }\end{array}$ \\
\hline $\begin{array}{c}f_{0}(500) \\
\text { veya } \sigma\end{array}$ & $\begin{array}{l}u \bar{d} \\
{[u d][\bar{u} \bar{d}]} \\
\pi \pi\end{array}$ & $0^{++}$ & $400-700$ & $\begin{array}{l}\text { Gluontopu } \\
{[58,59]} \\
\text { Tetrakuark } \\
{[23]} \\
\text { Molekül [23] }\end{array}$ & $\begin{array}{l}\text { E791[60] } \\
\text { BESIII [61] }\end{array}$ & $\begin{array}{c}\eta_{c} \rightarrow \eta \pi^{+} \pi^{-} \\
\chi_{c 1} \rightarrow \eta \pi^{+} \pi^{-}\end{array}$ \\
\hline $\begin{array}{l}\kappa \\
\text { veya } \\
K_{0}^{*}(800)\end{array}$ & $\begin{array}{l}u \overline{\mathrm{s}} \\
{[u d][\bar{d} \bar{s}]} \\
{[u d][\bar{u} \bar{s}]} \\
K \pi\end{array}$ & $0^{+}$ & $547 \pm 24$ & $\begin{array}{l}\text { Tetrakuark } \\
{[23]} \\
\text { Molekül [23] }\end{array}$ & E791 [60] & $D^{+} \rightarrow K^{-} \pi^{+} \pi^{-}$ \\
\hline$f_{0}(980)$ & 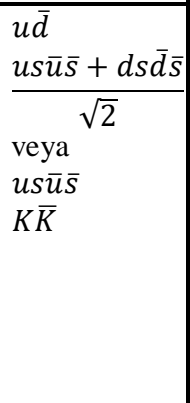 & $0^{++}$ & $10-100$ & $\begin{array}{l}\text { Gluontopu } \\
{[62]} \\
\text { Tetrakuark } \\
{[23,63]} \\
\text { Molekül } \\
{[23,64,65]} \\
a_{0}(980) \text { ile } \\
\text { karışım [66] }\end{array}$ & $\begin{array}{l}\text { E791 [60] } \\
\text { BESIII [62] } \\
\text { CLEO [67] } \\
\text { LHCb [68] } \\
\text { Belle [69] }\end{array}$ & $\begin{array}{l}D_{s}^{+} \rightarrow \pi^{+}\left[\pi^{+} \pi^{-}\right] \\
D_{s}^{+} \rightarrow \pi^{+}\left[K^{+} K^{-}\right] \\
e^{+} e^{-} \rightarrow \varphi \gamma f_{0}(980 \\
B \rightarrow f_{0}(980) K \\
B \rightarrow J / \psi f_{0}(980) \\
f_{0}(980) \rightarrow \pi^{+} \pi^{-}\end{array}$ \\
\hline$a_{0}(980)$ & $\begin{array}{l}u \bar{d} \\
{[d s][\bar{u} \bar{s}]} \\
\frac{u s \bar{u} \bar{s}-d s \bar{d} \bar{s}}{\sqrt{2}} \\
{[u s][\bar{d} \bar{s}]} \\
K \bar{K}\end{array}$ & $0^{++}$ & $50-100$ & $\begin{array}{l}\text { Tetrakuark } \\
{[23]} \\
\text { Molekül } \\
{[23,65]}\end{array}$ & $\begin{array}{l}\text { Belle [70] } \\
\text { BESIII [61] } \\
\text { COSY [71] } \\
\text { BaBar [72] } \\
\text { KLOE [73] }\end{array}$ & $\begin{array}{l}\eta_{c} \rightarrow \eta \pi^{+} \pi^{-} \\
\chi_{c 1} \rightarrow \eta \pi^{+} \pi^{-} \\
p p \rightarrow p p K^{+} K^{-} \\
D^{0} \rightarrow \bar{K}^{0} a_{0}(980), \\
\bar{K}^{0} \varphi \\
K^{-} a_{0}^{+}(980) \\
e^{+} e^{-} \rightarrow \varphi \\
\rightarrow \gamma a_{0}(980)\end{array}$ \\
\hline$f_{0}(1370)$ & $\bar{u} \bar{d}$ & $0^{++}$ & $200-500$ & $\begin{array}{l}\text { Tetrakuark } \\
{[74]} \\
\text { Gluontopu } \\
{[75]}\end{array}$ & $\begin{array}{l}\text { BESIII [61] } \\
\text { GAMs [76] } \\
\text { Crystal [77] } \\
\text { Barrel [78] } \\
\text { BaBar [79] }\end{array}$ & $\begin{array}{l}D_{S}^{+} \rightarrow \pi^{+} \pi^{+} \pi^{-} \\
D^{0} \rightarrow \sigma f_{0}(1370) \\
J / \psi \rightarrow \gamma f_{0}(1370) \\
\psi(2 S) \rightarrow \gamma f_{0}(1370) \\
\\
Y(1 S) \rightarrow \gamma f_{0}(1370) \\
Y(2 S) \rightarrow \gamma f_{0}(1370)\end{array}$ \\
\hline$\pi_{1}(1400)$ & $\begin{array}{l}u \bar{d} \\
\eta(1295) \pi\end{array}$ & $1^{-+}$ & $330 \pm 35$ & $\begin{array}{l}\text { Tetrakuark } \\
{[80]} \\
\text { Molekül [81] }\end{array}$ & $\begin{array}{l}\text { GAMs [82] } \\
\text { KEK [83] } \\
\text { Crystal } \\
\text { Barrel[84] } \\
\text { E852 [85] }\end{array}$ & $\begin{array}{l}\pi^{-} p \rightarrow\left(\eta \pi^{-}\right) p \\
p \bar{n} \rightarrow \pi^{-} \pi^{-} \pi^{0} \eta\end{array}$ \\
\hline$\Lambda(1405)$ & $\begin{array}{l}u d s \\
\bar{K} N(\mathrm{~S}) \\
\pi \Sigma \\
\bar{D}^{*} \Sigma_{c}\end{array}$ & $1 / 2^{-}$ & $50.5 \pm 2$ & $\begin{array}{l}\text { Hibrit [86] } \\
\text { Molekül } \\
{[87,88,89,90]}\end{array}$ & $\begin{array}{l}\text { Siddharta [91] } \\
\text { LEPS [92] } \\
\text { CLAS [93] }\end{array}$ & $\gamma p \rightarrow K^{+} \Lambda(1405)$ \\
\hline
\end{tabular}




\begin{tabular}{|c|c|c|c|c|c|c|}
\hline & & & & & HADES [94] & \\
\hline$K_{0}^{*}(1430)$ & $u \bar{s}$ & $0^{+}$ & $270 \pm 80$ & $\begin{array}{l}\text { Gluontopu } \\
\text { [95] }\end{array}$ & $\begin{array}{l}\text { CERN-USSR } \\
\text { [97] }\end{array}$ & $K^{+} p \rightarrow K^{*+}(1430) p$ \\
\hline$a_{0}(1450)$ & $u \bar{d}$ & $0^{++}$ & $265 \pm 13$ & $\begin{array}{l}\text { Gluontopu } \\
\text { [95] }\end{array}$ & $\begin{array}{l}\text { Crystal Barrel } \\
{[98,99]}\end{array}$ & $\bar{p} p \rightarrow \eta^{\prime} \pi^{0} \pi^{0}$ \\
\hline$f_{0}(1500)$ & $\overline{u \bar{d}}$ & $0^{++}$ & $109 \pm 7$ & $\begin{array}{l}\text { Gluontopu } \\
{[95,96]}\end{array}$ & $\begin{array}{l}\text { BES [100] } \\
\text { BESIII [101] }\end{array}$ & $\begin{array}{l}\bar{p} p \rightarrow \pi^{0} K_{L} K_{L} \\
J / \psi \rightarrow \gamma f_{0}(1500) \\
f_{0}(1500) \rightarrow \pi \pi, \\
K \bar{K}, \eta \eta, \eta \eta^{\prime} \\
J / \psi \rightarrow \gamma \eta \eta\end{array}$ \\
\hline$f_{2}(1565)$ & $\begin{array}{l}u \bar{d} \\
\rho \rho+\omega \omega\end{array}$ & $2^{++}$ & $134 \pm 8$ & $\begin{array}{l}\overline{p p} \\
\text { baryonyum } \\
{[40]} \\
\text { Molekül [102] }\end{array}$ & $\begin{array}{l}\text { Asterix [40] } \\
\text { Obelix [103] }\end{array}$ & $\begin{array}{l}\bar{p} p \rightarrow \pi^{-} \pi^{+} \pi^{0} \\
\bar{n} p \rightarrow \pi^{-} \pi^{+} \pi^{0}\end{array}$ \\
\hline$\overline{\pi_{1}(1600)}$ & $u \bar{d}$ & $1^{-+}$ & $241 \pm 40$ & Hibrit [104] & $\begin{array}{l}\text { CLEO [105] } \\
\text { COMPASS } \\
{[50]} \\
\text { E852[106] } \\
\text { VES [107] }\end{array}$ & $\begin{array}{l}\chi_{c 1} \rightarrow \eta^{\prime} \pi^{+} \pi^{-} \\
\pi^{-} p \rightarrow \pi^{+} \pi^{-} \pi^{-} \\
\pi^{-} p \rightarrow\left(\eta^{0} \pi^{-}\right) p \\
\pi^{-} p \rightarrow\left(\eta^{0} \pi^{0}\right) n\end{array}$ \\
\hline$\eta_{2}(1645)$ & $u \bar{d}$ & $2^{-+}$ & $181 \pm 11$ & Hibrit [108] & $\begin{array}{l}\text { Crystal Barrel } \\
{[109]}\end{array}$ & $\bar{p} p \rightarrow \pi^{0} \pi^{0} \pi^{0} \eta$ \\
\hline $\bar{\pi} \pi_{2}(1670)$ & $u \bar{d}$ & $2^{-+}$ & $260 \pm 9$ & Hibrit [110] & E852 [50] & $\pi^{-} p \rightarrow \pi^{+} \pi^{-} \pi^{-} p$ \\
\hline$f_{0}(1710)$ & $s \bar{s}$ & $0^{++}$ & $139 \pm 8$ & $\begin{array}{l}\text { Gluontopu } \\
{[111,112]}\end{array}$ & $\begin{array}{l}\text { GlueX [113] } \\
\text { BESIII [114] }\end{array}$ & $\begin{array}{l}\gamma \mathrm{p} \rightarrow \mathrm{p} \pi^{0} \\
\gamma \mathrm{p} \rightarrow \mathrm{p} \eta \\
J / \psi \rightarrow \gamma \eta \eta\end{array}$ \\
\hline$f_{0}(1810)$ & $u \bar{d}$ & $2^{++}$ & $105 \pm 20$ & $\begin{array}{l}\text { Gluontopu } \\
\text { [115] }\end{array}$ & $\begin{array}{l}\text { BESIII } \\
{[116,117]}\end{array}$ & $\begin{array}{l}J / \psi \rightarrow \gamma \eta \eta \\
J / \psi \rightarrow \gamma \pi^{0} \pi^{0}\end{array}$ \\
\hline$\pi(1800)$ & $u \bar{d}$ & $0^{-+}$ & $208 \pm 12$ & Hibrit [118] & $\begin{array}{l}\text { VES [118] } \\
\text { COMPASS } \\
{[119]}\end{array}$ & $\pi^{-} p \rightarrow \pi^{+} \pi^{-} \pi^{-} p$ \\
\hline$\eta_{2}(1870)$ & $u \bar{d}$ & $2^{-+}$ & $225 \pm 14$ & $\begin{array}{l}\text { Hibrit } \\
{[120,121]}\end{array}$ & WA102 [122] & $\begin{array}{l}p p \\
\rightarrow p_{f}\left(\pi^{+} \pi^{-} \pi^{+} \pi^{-}\right) p_{s}\end{array}$ \\
\hline$D_{s 0}^{*}(2317)^{ \pm}$ & $\begin{array}{l}c \bar{s} \\
{[c q][\bar{s} \bar{q}]} \\
c \bar{s} q \bar{q} \\
D K\end{array}$ & $0^{+}$ & $<3.8$ & $\begin{array}{l}\text { Molekül } \\
{[37,36,123]} \\
\text { Tetrakuark } \\
{[124,125]}\end{array}$ & $\begin{array}{l}\text { BaBar [126] } \\
\text { Belle [127] } \\
\text { CLEO [128] } \\
\text { Focus [129] }\end{array}$ & $\begin{array}{l}\overline{p p} \rightarrow D_{s}^{-} D_{s 0}^{*}(2317)^{+} \\
B \rightarrow D_{s 0}^{*}(2317)^{+} \underline{D}^{*} \\
e^{+} e^{-} \rightarrow D_{s}(2317) \\
\quad \rightarrow D_{s} \pi^{0}\end{array}$ \\
\hline$D_{s 1}(2460)^{ \pm}$ & $\begin{array}{l}c \bar{s} \\
D^{*} K\end{array}$ & $1^{+}$ & $<3.5$ & $\begin{array}{l}\text { Molekül [123] } \\
\text { Tetrakuark } \\
{[124,125]}\end{array}$ & $\begin{array}{l}\text { CLEO[128] } \\
\text { BaBar [126] } \\
\text { Belle [127] }\end{array}$ & $B \rightarrow D_{s 1}(2460)^{+} \bar{D}(*)$ \\
\hline$\Omega_{c}(3119)^{0}$ & $\begin{array}{l}c \bar{q} \\
s s c q \bar{q} \\
\Xi_{c} K \mathrm{ve} \\
\Xi_{c}^{\prime} K\end{array}$ & $3 / 2^{-}$ & $0.6-1.1$ & $\begin{array}{l}\text { Pentakuark } \\
{[130]} \\
\text { Molekül [131] }\end{array}$ & LHCb [132] & $\Xi_{c}^{+} \rightarrow p K^{-} \pi^{+}$ \\
\hline
\end{tabular}




\begin{tabular}{|c|c|c|c|c|c|c|}
\hline$X(3823)$ & $c \bar{c}$ & $2^{--}$ & $<16$ & Hibrit [133] & $\begin{array}{l}\text { Belle [134] } \\
\text { BESIII [135] } \\
\text { E705 [136] }\end{array}$ & $\begin{array}{l}B \rightarrow K X(3823)\left(\gamma \chi_{c 1}\right) \\
e^{+} e^{-} \rightarrow \pi^{+} \pi^{-} X(3823) \\
\psi^{\prime} \rightarrow J / \psi \pi^{+} \pi^{-}\end{array}$ \\
\hline$X(3872)$ & $\begin{array}{l}c \bar{c} \\
{[c q][\bar{c} \bar{q}]} \\
c \bar{c} q \bar{q} \\
D^{0} \bar{D}^{*^{0}}\end{array}$ & $\begin{array}{l}1^{++} \\
/ 2^{-+}\end{array}$ & $1.3 \pm 0.6$ & $\begin{array}{l}\text { Hibrit [137] } \\
\text { Molekül [138] } \\
\text { Tetrakuark } \\
{[139,140]}\end{array}$ & $\begin{array}{l}\text { Belle[4] } \\
\text { BaBar [141] } \\
\text { CDF[6], } \\
\text { DO[142] }\end{array}$ & $\begin{array}{l}B \rightarrow K X ; p \underline{p} \\
X \rightarrow \pi^{+} \pi^{-} J / \psi \\
X \rightarrow \pi^{+} \pi^{-} \pi^{0} J / \psi \\
X \rightarrow \gamma J / \psi ; \\
X \rightarrow \gamma \psi(2 S) \\
X(3875) \rightarrow D^{0} \bar{D}^{0} \pi^{0}\end{array}$ \\
\hline$Z_{c}(3900)^{0}$ & $q c \bar{q} \bar{c}$ & $1^{+-}$ & $29.6 \pm 8.2$ & $\begin{array}{l}\text { Tetrakuark } \\
\text { [143] }\end{array}$ & $\begin{array}{l}\text { BESIII [144] } \\
\text { CLEO-c [145] }\end{array}$ & $\begin{aligned} e^{+} e^{-} & \rightarrow \pi^{0} Z_{c}(3900) \\
& \rightarrow \pi^{0} \pi^{0} J / \psi\end{aligned}$ \\
\hline$Z_{c}(3900)^{+}$ & $\begin{array}{l}q c \bar{q} \bar{c} \\
D \bar{D}^{*}\end{array}$ & $1^{+-}$ & $35 \pm 7$ & $\begin{array}{l}\text { Tetrakuark } \\
{[146]} \\
\text { Molekül [147] }\end{array}$ & $\begin{array}{l}\text { BESIII [31] } \\
\text { Belle [148] }\end{array}$ & $\begin{array}{l}e^{+} e^{-} \rightarrow \pi^{ \pm} Z_{c}^{ \pm} \\
Z_{c}^{ \pm} \rightarrow \pi^{ \pm} J / \psi \\
Z_{c} \rightarrow h_{c} \pi \\
e^{+} e^{-} \rightarrow \psi(4160) \\
\rightarrow \pi^{ \pm} J / \psi\end{array}$ \\
\hline$X(3915)$ & $\begin{array}{l}c \bar{c} \\
D \bar{D}^{*}\end{array}$ & $\begin{array}{l}0^{++} \\
/ 2^{++}\end{array}$ & $20 \pm 5$ & Molekül [2] & $\begin{array}{l}\text { Belle [32], } \\
\text { BaBar [149] }\end{array}$ & $\begin{array}{l}B \rightarrow K(J / \psi \omega) \\
e^{+} e^{-} \rightarrow e^{+} e^{-} \\
\gamma \gamma \rightarrow J / \psi \omega\end{array}$ \\
\hline $\begin{array}{c}X(3940) \\
\text { veya } \\
Y(3940) \\
\end{array}$ & $\begin{array}{l}c \bar{c} \\
D \bar{D}^{*}\end{array}$ & $?^{?+}$ & $37_{-17}^{+27}$ & $\begin{array}{l}\text { Hibrit }[150] \\
\text { Molekül } \\
{[151,152]}\end{array}$ & $\begin{array}{l}\text { Belle[153] } \\
\text { BaBar [154] }\end{array}$ & $\begin{aligned} & e^{+} e^{-} \rightarrow J / \psi Y \\
& \rightarrow J / \psi\left(D \bar{D}^{*}\right) \\
& B \rightarrow K Y(3940) \\
& Y \rightarrow J / \psi \omega\end{aligned}$ \\
\hline$Z_{c}(4020)^{ \pm}$ & $\begin{array}{l}s c \bar{s} \bar{c} \\
D \bar{D}^{*}\end{array}$ & $\begin{array}{l}1^{+-} \\
/ 2^{+}\end{array}$ & $7.9 \pm 3.7$ & $\begin{array}{l}\text { Tetrakuark } \\
{[155]} \\
\text { Molekül [147] }\end{array}$ & $\begin{array}{l}\text { Belle [156] } \\
\text { BESIII [157] }\end{array}$ & $\begin{array}{l}e^{+} e^{-} \rightarrow \pi\left[D \bar{D}^{*}\right] \\
e^{+} e^{-} \rightarrow \pi\left[h_{c} \pi\right] \\
e^{+} e^{-} \rightarrow \pi\left[\psi^{\prime} \pi\right]\end{array}$ \\
\hline $\begin{array}{l}X(4140) \\
\text { veya } \\
\mathrm{Y}(4140)\end{array}$ & $\begin{array}{l}c \bar{c} \\
c \bar{c} s \bar{S} \\
s c \bar{S} \bar{c} \\
D_{S}^{*} \bar{D}_{S}^{*}\end{array}$ & $1^{++}$ & $15_{-5}^{+6}$ & $\begin{array}{l}\text { Hibrit }[158] \\
\text { Molekül } \\
{[159,160]} \\
\text { Tetrakuark } \\
{[161,162]}\end{array}$ & $\begin{array}{l}\text { CDF [163] } \\
\text { CMS [164] } \\
\text { DO [165] } \\
\text { LHCb [33] } \\
\text { Babar [166] }\end{array}$ & $\begin{aligned} p \bar{p} \rightarrow B^{+} & \rightarrow Y(4140) K^{+} \\
& \rightarrow K[J / \psi \phi] \\
B & \rightarrow J / \psi K K K\end{aligned}$ \\
\hline$Y(4220)$ & $\begin{array}{l}c \bar{c} g \\
D \bar{D}(2420)\end{array}$ & $\overline{1^{--}}$ & $\begin{array}{l}44.1 \pm 4.3 \\
0.5 \mathrm{ve} \\
0.023\end{array}$ & $\begin{array}{l}\text { Hibrit [167] } \\
\text { Molekül [168] } \\
\text { Tetrakuark } \\
{[169]}\end{array}$ & $\begin{array}{l}\text { Belle }[170] \\
\text { BESIII }[8,171]\end{array}$ & $\begin{array}{l}e^{+} e^{-} \rightarrow \pi^{+} \pi^{-} \psi(2 S) \\
e^{+} e^{-} \rightarrow \pi^{+} \pi^{-} h_{c} \\
e^{+} e^{-} \rightarrow \omega \chi_{c J}\end{array}$ \\
\hline$Y(4260)$ & $\begin{array}{l}c \bar{c} g \\
{[c q][\bar{c} \bar{q}]} \\
\bar{D} D_{1}(2420) \\
\text { veya } \\
D_{0} \bar{D}^{*} \\
\Lambda_{c} \bar{\Lambda}_{c} \\
\text { baryonyum }\end{array}$ & $1^{--}$ & $120 \pm 12$ & $\begin{array}{l}\text { Hibrit [172] } \\
\text { Molekül [168] } \\
\text { Tetrakuark } \\
{[25]} \\
\text { Baryonyum } \\
{[173]}\end{array}$ & $\begin{array}{l}\text { BaBar [174] } \\
\text { Cleo [175] } \\
\text { BESIII [31] } \\
\text { Belle [176] }\end{array}$ & $\begin{array}{l}e^{+} e^{-} \rightarrow \pi^{+} \pi^{-} J / \psi \\
e^{+} e^{-} \rightarrow \pi D \bar{D}^{*} \\
e^{+} e^{-} \rightarrow \chi_{c 0} \omega\end{array}$ \\
\hline
\end{tabular}




\begin{tabular}{|c|c|c|c|c|c|c|}
\hline$Y(4360)$ & {$[c q][\bar{c} \bar{q}]$} & $1^{--}$ & $74 \pm 18$ & $\begin{array}{l}\text { Tetrakuark } \\
{[155,25]}\end{array}$ & $\begin{array}{l}\text { BaBar [177] } \\
\text { Belle [178] }\end{array}$ & $\begin{array}{l}e^{+} e^{-} \\
\rightarrow \gamma \pi^{+} \pi^{-} \psi(3686)\end{array}$ \\
\hline$P_{c}(4380)^{+}$ & $\begin{array}{l}c \bar{c} u u d \\
\bar{D}^{*} \Sigma_{c}\end{array}$ & $3 / 2^{+}$ & $205 \pm 90$ & $\begin{array}{l}\text { Pentakuark } \\
{[7]} \\
\text { Molekül } \\
{[179,180]}\end{array}$ & $\overline{\mathrm{LHCb}}[7]$ & $\Lambda_{b}^{0} \rightarrow J / \psi p K^{-}$ \\
\hline$Y(4390)$ & $D^{*} \bar{D}_{0}^{*}(2400)$ & $\overline{1^{--}}$ & $\begin{array}{l}153.2 \\
\pm 11.4 \\
\end{array}$ & Molekül [168] & BESIII [39] & $e^{+} e^{-} \rightarrow \gamma \pi^{+} \pi^{-} h_{c}$ \\
\hline$Z_{c}(4430)^{ \pm}$ & $\begin{array}{l}c \bar{c} u \bar{d} \\
D_{1} \bar{D}^{*} \\
\text { veya } \\
D_{2} \bar{D}^{*}\end{array}$ & $1^{+}$ & $200_{-58}^{+49}$ & $\begin{array}{l}\text { Molekül [29] } \\
\text { Tetrakuark } \\
{[181]}\end{array}$ & $\begin{array}{l}\text { Belle [29, 182] } \\
\text { BaBar [29] } \\
\text { LHCb [30] }\end{array}$ & $\begin{array}{l}\bar{B}^{-, 0} \rightarrow K^{0,+}\left[J / \psi \pi^{-}\right] \\
B \rightarrow K\left[\pi^{+} J / \psi\right] \\
B \rightarrow K Z \rightarrow K^{+}\left[\psi^{\prime} \pi^{ \pm}\right]\end{array}$ \\
\hline$P_{c}(4450)^{+}$ & $\begin{array}{c}c \bar{c} u u d \\
\bar{D}^{*} \Sigma_{c}(S) \\
\text { ve } \\
\bar{D}^{*} \Lambda_{c} \\
\text { karıșımı } \\
\end{array}$ & $5 / 2^{+}$ & $39 \pm 20$ & $\begin{array}{l}\text { Pentakuark } \\
\text { [7] } \\
\text { Molekül [180] }\end{array}$ & $\overline{\mathrm{LHCb}[7]}$ & $\Lambda_{b}^{0} \rightarrow J / \psi p K^{-}$ \\
\hline$X(4500)$ & $\begin{array}{l}c \bar{c} \\
c s \bar{c} \bar{S}\end{array}$ & $0^{++}$ & $92 \pm 21_{-20}^{+21}$ & $\begin{array}{l}\text { Tetrakuark } \\
{[183]}\end{array}$ & LHCb [184] & $B^{+} \rightarrow K^{+}[J / \psi \phi]$ \\
\hline $\begin{array}{l}X(4660) \\
\text { veya } \\
Y(4660)\end{array}$ & $\begin{array}{l}c \bar{c} \\
c \bar{c} g \\
\bar{c} c \bar{q} q \\
\psi^{\prime} f_{0}(980)\end{array}$ & $\overline{1^{--}}$ & $48 \pm 15$ & $\begin{array}{l}\text { Hibrit [185] } \\
\text { Molekül [186] } \\
\text { Tetrakuark } \\
\text { [155] }\end{array}$ & $\begin{array}{l}\text { Belle [187] } \\
\text { BaBar [178] }\end{array}$ & $e^{+} e^{-} \rightarrow \pi^{+} \pi^{-} \psi(2 \mathrm{~S})$ \\
\hline$X(4700)$ & $\begin{array}{l}c \bar{c} \\
c s \bar{c} \bar{s}\end{array}$ & $0^{++}$ & $\begin{array}{l}120 \\
\pm 31_{-33}^{+42}\end{array}$ & $\begin{array}{l}\text { Tetrakuark } \\
\text { [183] }\end{array}$ & LHCb [33] & $B^{+} \rightarrow K^{+}[J / \psi \phi]$ \\
\hline$X(5568)$ & $\begin{array}{c}{[b u][\bar{d} \bar{s}} \\
{[b d][\bar{s} \bar{u}]} \\
{[s u][\bar{b} \bar{d}]} \\
\text { veya } \\
{[s d][\bar{b} \bar{u}]} \\
s \bar{b} u \bar{d} \\
B \bar{K} \text { veya } \\
B^{*} \bar{K} \\
\end{array}$ & $0^{+}$ & $21.9 \pm 6.4$ & $\begin{array}{l}\text { Molekül } \\
{[188,189]} \\
\text { Tetrakuark } \\
{[190]}\end{array}$ & $\begin{array}{l}\text { LHCb [35] } \\
\text { D0 [34] }\end{array}$ & $\begin{array}{l}X(5568) \rightarrow B_{s}^{0} \pi^{+} \\
B_{s}^{0} \rightarrow J / \psi \phi \\
J / \psi \rightarrow \mu^{+} \mu^{-} \\
\phi \rightarrow K^{+} K^{-}\end{array}$ \\
\hline$Z_{b}(10610)^{0}$ & $\begin{array}{l}b \bar{b} u \bar{d} \\
B^{*} \bar{B}^{*}\end{array}$ & $1^{+-}$ & $?$ & & Belle [191] & $e^{+} e^{-} \rightarrow\left(\Upsilon(2,3 S) \pi^{0}\right) \pi^{0}$ \\
\hline$Z_{b}(10610)^{+}$ & $\begin{array}{l}b \bar{b} u \bar{d} \\
B^{*} \bar{B}^{*}\end{array}$ & $\begin{array}{l}1^{+-} \\
1^{++} \\
/ 0^{++} \\
/ 2^{++}\end{array}$ & $18.4 \pm 2.4$ & $\begin{array}{l}\text { Molekül [147] } \\
\text { Tetrakuark } \\
{[192,193]}\end{array}$ & Belle [194] & $\begin{array}{c}e^{+} e^{-} \rightarrow Y(10860) \\
\rightarrow \pi\left[B^{*} \bar{B}^{*}\right] \\
Y(10860) \rightarrow \pi[Y \pi] \\
Y(10860) \rightarrow \pi\left[h_{b} \pi\right]\end{array}$ \\
\hline
\end{tabular}

III. SONUÇ

1964'te Murray Gell-Mann ve George Zweig tarafindan önerilen ve hadronların sınıflandırılması 
için en etkili model olan Kuark Modeli 2000'li yıllara kadar deneylerde gözlemlenen parçacıkların özelliklerini büyük ölçüde başarılı bir şekilde açıkladı. Bu modele göre mezonlar bir kuarkantikuark çiftinden $(q \bar{q})$, baryonlar ise üç kuarktan (qqq) oluşmaktadırlar. Güçlü etkileşimin kuramı Kuantum Renk Dinamiğine göre Standart Kuark Modelinin dışında farklı yapılara sahip hadronlar olabilirdi. KRD'ye göre hadron spektrumu geleneksel Kuark Modelinin öngördüğü parçacık spektrumundan çok daha zengin ve geniş yapıya sahiptir. Standart Kuark Modelinin gözlemlenen tüm hadronların özelliklerini başarılı bir şekilde açıklayabilmesi Modelin dışında hadron yapılarının mevcut olamayacağ 1 düşüncesini ortaya çıkardı. Uzun yillar yüksek enerji fiziğinde kabul gören bu yaklaşım bazı fizikçiler tarafindan zaman zaman sorgulaniyordu. 1975 'de Fritzsch ve Minkowski yalnız gluonlardan oluşabilecek ve gluontopu olarak adlandırılan durumlarının var olabileceğini, 1977 yılinda

Jaffe $a_{0}(980), f_{0}(980)$ ve $\sigma$ parçacıklarının

bilinen parçacık yapısında olmadığını ve bu parçacıkların tetrakuark yapıda ([qq] $[q \bar{q}])$ olabileceğini öne sürdü. Ayrıca, 1990'ların sonunda bazı yüksek enerji fizikçileri tarafindan dört kuark ve bir antikuarktan oluşan baryonların (pentakuarklar) var olabileceği öne sürüldü.

XX. yüzyılın sonu ve XXI. yüzyılın başlangıcında hızlandırıcılarda gözlemlenen birçok parçacık standart kuark modeli çerçevesinde açıklanamayan özellikler gösteriyordu. 2003 yılında Japonya'daki Belle deneyinde gözlemlenen ve dört kuark içerdiği öngörülen $X(3872), \quad 2007$ 'de keşfedilen $Z(4430)$ ve $Y(4660), 2009$ yılında keşfedilen X(4140), 2014 yilında LHC'de gözlemlenen $X(4274), X(4500), \quad X(4700)$ parçacıkları ve çok sayıda diğer parçacık geleneksel Kuark Modelinin yetersizliğini gösteriyordu. Standart olmayan doğalarından dolayı, bu parçacıklara "egzotik parçacıklar" adı verildi.

$\mathrm{Bu}$ parçacıkların keşfi hadron spektroskopisinde yeni bir çağın başladığını göstermektedir. Son yıllarda egzotik parçacıklarla ilgili çok sayıda deneysel ve teorik çalışmalar yapılmış ve bu araştırmalara devam edilmektedir. Günümüz yüksek enerji fizikçileri gluontopu (glueball) veya gluonyum, hibrit ( $q \bar{q} g$ veya $q q q g$ ), mezon molekül $(q q \bar{q} \bar{q})$, tetrakuark $(q \bar{q} q \bar{q})$, pentakuark $(q q q q \bar{q})$, hegzakuark ( $q q q q q q$ veya $\bar{q} \bar{q} \bar{q} \bar{q} \bar{q} \bar{q})$ ve dibaryon $(q q q \bar{q} \bar{q} \bar{q}) \quad$ yapıların incelenmesinin güçlü etkileşimin özelliklerinin ve kuarkların ebedi hapsolma olayının anlaşılmasına, Standart Model Ötesi fiziğin gelişimine büyük katkı sağlayacağını düşünmektedirler. Ayrıca, egzotik parçacıkların incelenmesi evrenin oluşumu, karanlık madde, karanlık enerji, nötron yıldızlarının içyapısının araştırılması bakımından da oldukça önemlidir.
Egzotik parçacıklar evrenin oluşumunda önemli bir rol oynamış olabilirler. Bir nötron yıldızının geleneksel modeli, nötronlardan oluşmasıdır ve genellikle bir nötron yıldızı içindeki parçacık etkileşmeleri nötronlar arasında olur. Nötron yıldızının çekirdeğinde nötronlar güçlü bir şekilde etkileşerek, tetrakuarkları oluşturabilir. Bu durum pentakuarkların ve hegzakuarkların üretimini de sağlayabilir. Egzotik parçacıkların doğrulanması astrofizikçilerin, nötron yıldızlarının içyapısı hakkındaki bazı varsayımları yeniden gözden geçirmesine neden olabilir.

Kuark-gluon hibritleri, tetrakuark durumları, hadron molekülleri veya daha farklı egzotik durumların varlığının LHC ve diğer hızlandırıcılarda yapılacak yeni ölçümlerle netleştirilmesi gerekiyor. Önümüzdeki yıllarda yapılacak BESIII deneylerinde egzotik hadronların içyapılarının ve özelliklerinin iyi anlaşılması hedeflenmektedir. 2019 yılında GSI'daki PANDA deneyinde antiprotonlar kullanarak, tilsımlı hibrit durumlarının araştırtırılması planlanmaktadır. FAIR deneyindeki PANDA dedektörünün kütle çözünürlüğü $B$ fabrikalarından yirmi kat daha fazla olacak şekilde tasarlanmaktadır. Elde edilecek yeni deney verilerinin analizi hadron spektroskopisi ile ilgili çok daha net bilgilere ulaşılmasını sağlayacaktır.

\section{KAYNAKLAR}

[1] Gell-Mann, M. (1964). A schematic model of baryons and mesons. Phys. Lett. 8, 214.

[2] Olive, K.A. et al. [Particle Data Group] (2015). Non$q \bar{q}$ mesons. Chin. Phys. C, 38, 090001.

[3] Besson, D. et al., [CLEO Collaboration] (2003). Observation of a narrow resonance of mass $2.46 \mathrm{GeV} / c^{2}$ decaying to $D_{S}^{*+} \pi^{0}$ and confirmation of the $D_{S J}^{*}$ (2317) state, Phys. Rev. D, 68, 032002, [Erratum: Phys. Rev. D75, 119908 (2007)].

[4] Choi, S.K. et al., [Belle Collaboration] (2003). Observation of a new narrow charmonium state in exclusive $B^{ \pm} \rightarrow K^{ \pm} \pi^{+} \pi^{-} J / \psi$ decays, Phys. Rev. Lett. 91, 262001 .

[5] Abazov, V. M. et al., [D0 Collaboration] (2016). Evidence for a $B_{S}^{0} \pi^{ \pm}$state, Phys.Rev.Lett., 117 (2) 022003 .

[6] Acosta, D. et al. [CDF II Collaboration] (2004). Observation of the narrow state $X(3872) \rightarrow J / \psi \pi^{+} \pi^{-}$in $\bar{p} p$ collisions at $\sqrt{s}=1.96 \mathrm{TeV}$, Phys.Rev.Lett. 93, 072001 .

[7] Aaij, R. et al. [LHCb Collaboration] (2015). Observation of $J / \psi p$ resonances consistent with pentaquark states $\Lambda_{b}^{0} \rightarrow J / \psi K^{-} p$ decays. Phys.Rev.Lett. $115,072001$. 
[8] Ablikim, M. et al. [BESIII Collaboration] (2017). Precise measurement of the $e^{+} e^{-} \rightarrow \pi^{+} \pi^{-} J / \psi$ cross section at center- of- mass energies from 3.77 to $4.60 \mathrm{GeV}$. Phys.Rev.Lett. 118, 092001.

[9] Chen, H. et. al. (2017). A review of the open charm and open bottom systems, Reports on Progress in Physics, 80, 7.

[10] Brambilla, N. et al. (2011). Heavy quarkonium: progress, puzzles, and opportunities. Eur. Phys. J. C 71, 1534.

\section{[11] https://en.wikipedia.org}

[12] Fritzsch, H. and Gell-Mann, M. (1972). Current algebra: Quarks and what else? eConf, 11 C720906V2, 135-165.

[13] Fritzsch, H. and Minkowski, P. (1975). Psi resonances, gluons and the Zweig Rule, Nuovo Cim. A 30, 393.

[14] Dzierba, A. R., Meyer, C. A. and Swanson, E. S. (2000). The search for QCD exotics. Am.Sci. 88, 406-415.

[15] Robson, D. (1977). A basic guide for the glueball spotter. Nucl.Phys. B, 130, 328-348.

[16] https://en.wikipedia.org/wiki/Glueball

[17] Klempt, E and Zaitsev, A. (2007). Glueballs, hybrids, multiquarks. Experimental facts versus QCD inspired concepts. Phys.Rept. 454, 1-202.

[18] Lee, W. and Weingarten, D. (2000). Scalar quarkonium masses and mixing with the lightest scalar glueball. Phys.Rev. D 61, 014015.

[19] Bali, G.S. et al. [UKQCD Collaboration] (1993). A Comprehensive lattice study of $S U(3)$ glueballs. Phys. Lett. B 309, 378.

[20] Morningstar, C.J. and Peardon, M.J. (1997). Efficient glueball simulations on anisotropic lattices. Phys. Rev. D 56,4043 .

[21] Chen, Y. et al. (2006). Glueball spectrum and matrix elements on anisotropic lattices. Phys.Rev. D 73, 014516.

[22] Loan, M. and Ying, Y. (2006). Sizes of lightest glueballs in $S U(3)$ lattice gauge theory. Prog. Theor. Phys. $116,169$.

[23] Jaffe, R.L. (1977). Perhaps a Stable Dihyperon? Phys.Rev. Lett. 38 (5) 195.

[24] Swanson, E.S. (2006). The New heavy mesons: A status report, Phys.Rept. 429, 243-305.

[25] Chen, H.X. et al. (2016). The hidden-charm pentaquark and tetraquark states. Phys.Rept. 639, 1-121.
[26] Cotugno, G., Faccini, R., Polosa, A.D. and Sabelli, C. (2010). Charmed baryonium. Phys.Rev.Lett. 104 (13) 132005 .

[27] T. Aaltonen et al. [CDF Collaboration] (2009). Evidence for a narrow near-threshold structure in the $J / \psi \phi$ mass spectrum in $B^{+} \rightarrow J / \psi \phi K^{+}$decays. Phys.Rev.Lett. 102, 242002.

[28] Ali, A., Hambrock, C. and Aslam, M.J. (2010). Tetraquark interpretation of the BELLE data on the anomalous $\quad \gamma(1 S) \pi^{+} \pi^{-}$and $\gamma(2 S) \pi^{+} \pi^{-}$production near the $\Upsilon(5 S)$ resonance. Phys.Rev.Lett. 104 (16) 162001.

[29] Choi, S.K. et al. [Belle Collaboration] (2008). Observation of a resonance-like structure in the $\pi^{ \pm} \psi^{\prime}$ mass distribution in exclusive $B \rightarrow K \pi^{ \pm} \psi^{\prime}$ decays. Phys.Rev. Lett. 100, 142001.

[30] Aaij, R. et al. [LHCb collaboration] (2014). Observation of the resonant character of the $Z(4430)$ state. Phys.Rev.Lett. 112, 222002.

[31] Ablikim, M. et al. [BESIII collaboration] (2013). Observation of a charged charmoniumlike structure in $e^{+} e^{-} \rightarrow \pi^{+} \pi^{-} \mathrm{J} / \psi$ at $\sqrt{s}=4.26 \mathrm{GeV}$. Phys.Rev.Lett. 110 (25) 252001 .

[32] Liu, Z. et al. [Belle collaboration] (2013). Study of $e^{+} e^{-} \rightarrow \pi^{+} \pi^{-} J / \psi$ and Observation of a charged charmoniumlike state at Belle, Phys.Rev.Lett. 110 (25) 252002 .

[33] Aaij, R. et al. [LHCb Collaboration] (2017). Observation of $J / \psi \varphi$ structures consistent with exotic states from amplitude analysis of $B^{+} \rightarrow J / \psi \phi K^{+}$decays. Phys Rev Lett. 118, 022003.

[34] Abazov, V.M. et al.(2016). Evidence for a $B_{s}^{0} \pi^{ \pm}$state D0 Collaboration. Phys.Rev.Lett. 117 (2) 022003.

[35] Aaij, R. et al. [LHCb Collaboration] (2016). Search for structure in the $B_{s}^{0} \pi \pm$ invariant mass spectrum Phys.Rev.Lett. 117 (15) 152003, Addendum: Phys.Rev.Lett. 118 (2017) no.10, 109904.

[36] Barnes, T. et al. (2003). Implications of a DK molecule at $2.32 \mathrm{GeV}$. Phys.Rev. D68, 054006.

[37] Liu, L. et al. (2013). Interactions of charmed mesons with light pseudoscalar mesons from lattice QCD and implications on the nature of the $D_{s 0}^{*}(2317)$. Phys.Rev. $D$ 87, 014508 .

[38] Guo, F.K., et al. (2017). Hadronic molecules. e-Print: arXiv:1705.00141 [hep-ph].

[39] Ablikim, M. et al. [BESIII Collaboration] (2017). Evidence of two resonant structures in $e^{+} e^{-} \rightarrow \pi^{+} \pi^{-} h_{c}$, Phys.Rev.Lett. 118, 092002.

[40] May, B. et al. [ASTERIX Collaboration] (1990). Anti-proton proton annihilation at rest in $H_{2}$ gas into $\pi^{+} \pi^{-} \pi^{0}$. Z. Phys. C46, 203. 
[41] Bertin, A. et al. (1998). Study of the $f_{0}$ (1500)/ $f_{0}(1565)$ production in the exclusive annihilation $\bar{n} p \rightarrow$ $\pi^{+} \pi^{+} \pi^{-}$n flight. Phys.Rev. D 57, 55.

[42] Pakhlova, G. et al. [Belle Collaboration] (2008). Observation of a near-threshold enhancement in the $e^{+} e^{-} \rightarrow \Lambda_{b} \Lambda_{c} \quad$ cross section using initial-state radiation. Phys.Rev. Lett. 101, 172001.

[43] Cotugno, G. et al. (2010). Charmed baryonium. Phys.Rev.Lett. 104, 132005.

[44] Guo, F.K. et al. (2010). Reconciling the $X$ (4630) with the $Y$ (4660). Phys.Rev. D 82, 094008.

[45] Adlarson, P. et al. [WASA-at-COSY Collaboration] (2015). Measurement of the $n p \rightarrow n p \pi^{0} \pi^{0}$ reaction in search for the recently observed $d *(2380)$ resonance. Phys.Lett. B 74, 325-332.

[46] Lacock, P. et al. (1997). Hybrid mesons from quenched QCD. Phys.Lett. B 401, 308.

[47] Bernard, C. et al. (1997). Exotic mesons in quenched lattice QCD. Phys.Rev. D 56, 7039.

[48] Kuhn, J. (2004). Evidence for exotic mesons. AIP Conf.Proc. 717, 377-386.

[49] Lu, M. et al. [E852 Collaboration] (2005). Exotic meson decay to $\omega \pi^{0} \pi^{-}$. Phys.Rev.Lett. 94, 032002.

[50] Chung, S.U. (2015). Recent COMPASS results and future prospects for ALICE Published in AIP Conf.Proc. 1654, 050009.

[51] Alekseev, M.G. et al. (2010). Observation of a $J^{P C}=$ $1^{-+}$exotic resonance in diffractive dissociation of $190 \mathrm{GeV} / \mathrm{c} \pi^{-}$into $\pi^{-} \pi^{-} \pi^{+}$Phys.Rev.Lett. 104, 241803.

[52] Amelin, D.V. et al. [VES Collaboration] (1995). Study of resonance production in diffractive reaction $\pi^{-} A \rightarrow \pi^{+} \pi^{-} \pi^{-} A$. Phys.Lett. B 356, 595.

[53] Diakonov, D., Petrov V. and Polyakov, M. (1997). Exotic anti-decuplet of baryons: prediction from chiral solitons. Zeitschrift für Physik A Hadrons and Nuclei, 359 (3) 305-314.

[54] Nakano, T. et al. [LEPS Collaboration] (2009). Evidence of the $\Theta^{+}$in the $\gamma d \rightarrow K^{+} K^{-}$pn reaction. Phys. Rev. C. 79 (2) 025210.

[55] astr.gsu.edu/hbase/Particles/pquark.htm

[56] Zavertyaev, M. [WA89 and COMPASS Collaborations] (2005). A search for pentaquark candidates in experiments WA89 and COMPASS. Nucl. Phys. A 755, 387-390.

[57] Aubert, B. et al. [BaBar Collaboration] (2006). Search for the charmed pentaquark candidate $\theta_{c}(3100)^{0}$ ine $e^{+} e^{-}$annihilations at $\sqrt{s}=10.58 \mathrm{GeV}$. Phys.Rev. D 73, 091101.

[58] Mennessier, G., Narison, S. and Wang, X. G. (2010). The $\sigma$ and $f_{0}(980)$ from $K_{e 4} \oplus \pi \pi$ scatterings data Phys.Lett. B 688, 59.

[59] Ochs, W. (2013). The Status of glueballs. J.Phys.G 40, 043001 .

[60] Aitala, E.M. et al. [E791 Collaboration] (2001). Experimental evidence for a light and broad scalar resonance in $D^{+} \rightarrow \pi^{-} \pi^{+} \pi^{+}$decay. Phys.Rev.Lett. 86 770-774.

[61] M. Ablikim et al. [BESIII Collaboration] (2017). Amplitude analysis of the $\chi_{c 1} \rightarrow \eta \pi^{+} \pi^{-}$decays. Phys.Rev. D95 (3) 032002.

[62] Morningstar, C.J. and Peardon, M.J. (1999). The Glueball spectrum from an anisotropic lattice study. Phys.Rev. D 60, 034509.

[63] Achasov, N.N. (2000). On nature of scalar $a_{0}$ (980) and $f_{0}$ (980)mesons. Nucl.Phys. A675, 279C-284C.

[64] Oller, J.A. et al. (2003). Finite width effects in $\phi$ radiative decays. Nucl.Phys. A714, 161.

[65] Sekihara, T., Kumano, S. (2015). Constraint on $\bar{K} K$ compositeness of the $a_{0}(980)$ and $f_{0}(980)$ resonances from their mixing intensity. Phys.Rev. D92 (3) 034010.

[66] Ablikim, M. et al. [BESIII Collaboration] (2015). Observation of the isospin-violating decay $J / \psi \rightarrow \phi \pi^{0}$ $f_{0}$ (980). Phys.Rev. D 92 (1) 012007.

[67] Hietala, J., Cronin-Hennessy, D., Pedlar, T. and Shipsey, I. (2015). Exclusive $D_{S}$ semileptonic branching fraction measurements. Phys.Rev. D92 (1) 012009.

[68] Aaij, R. et al. [LHCb Collaboration]. (2015). Study of the rare $B_{S}^{0}$ and $B^{0}$ decays into the $\pi^{-} \pi^{+} \mu^{+} \mu^{-}$final state. Phys.Lett.B 743, 46.

[69] Li, J. et al. [Belle Collaboration] (2011). Observation of $B_{s}^{0} \rightarrow J / \psi f_{0}(980)$ and evidence for $B_{s}^{0} \rightarrow J /$ $\psi f_{0}(1370)$. Phys.Rev.Lett. 106, 121802.

[70] Uehara, A. (2009). High-statistics study of $\eta \pi^{0}$ production in two-photon collisions. Phys.Rev. D80, 032001 .

[71] Silarski, M. and Moskal, P. for the COSY-11 collaboration. (2010). Study of the near threshold $p p \rightarrow$ $p p K^{+} K^{-}$reaction in view of the $K^{+} K^{-}$final state interaction. Invited talk at the Excited QCD 2010 Workshop, 31 January-6 February, Stara Lesna, Slovakia.

[72] Sanchez, P. del Amo. et al. [BaBar Collaboration] (2010). Measurement of $D^{0}-\bar{D}^{0}$ mixing parameters using $D^{0} \rightarrow K_{S}^{0} \pi^{+} \pi^{-} \quad$ and $\quad D^{0} \rightarrow K_{S}^{0} K^{+} K^{-}$decays. Phys.Rev.Lett. 105, 081803. 
[73] Achasov, N.N. [KLOE Collaboration] (2003). Radiative decays of $\phi$ meson about nature of light scalar resonances. Nucl. Phys. A728, 425.

[74] Mukherjee, T.K., et al. (2014). Chiral phase transition with mixing between scalar quarkonium and tetraquark. Phys.Rev. D89 (7) 076002.

[75] Gutsche, T. et al. (2017). Role of scalar mesons in the beam asymmetry of $p \bar{p}$ and $\Lambda \bar{\Lambda}$ photoproduction at JLab. arXiv:1705.07002 [hep-ph].

[76] Alde et al., D. [GAMS Collaboration] (1999). New results of the GAMS collaboration on meson spectroscopy. Phys.Atom.Nucl. 62, 1993-1998, Yad.Fiz. 62 (1999) 21602166.

[77] Abele, et al. [Crystal Barrel Collaboration] (1996). Observation of $f_{0}(1500)$ decay into $K_{L} K_{L}$. Phys.Lett.B 385,425 .

[78] Abe, K. et al. [Belle Collaboration] (2002). Study of three-body charmless $B$ decays Phys.Rev. D65, 092005.

[79] Aubert, B. et al. [BABAR Collaboration] (2004). Measurements of the branching fractions of charged $B$ decays to $K^{ \pm} \pi^{\mp} \pi^{ \pm}$final states. Phys.Rev. D70, 092001.

[80] Chung, S.U., Klempt, E. and Korner, J.G. (2002). SU(3) classification of p-wave $\eta \pi$ and $\pi$ systems. Eur. Phys. J. A 15, 539

[81] Zhang, R., Ding, Y.B., Li, X.Q. and Page, P.R. (2002). Molecular states and $1^{-+}$exotic mesons. Phys.Rev. D 65 , 096005 .

[82] Alde, D. et al. [IHEP-IISN-LANL-LAPP Collaboration] (1988). Evidence for a $1^{-+}$exotic meson. Phys.Lett.B 205, 397.

[83] Aoyagi, H. et al. Study of the $\eta \pi^{-}$system in the $\pi$ $p$ reaction at $6.3 \mathrm{GeV} / c$. Phys.Lett. B314, 246 (1993).

[84] Abele, A. et al. [Crystal Barrel Collaboration] (1998). Exotic $\eta \pi$ state in $\bar{p} d$ annihilation at rest into $\pi-\pi^{0} \eta \mathrm{p}$ (spectator). Phys.Lett. B423, 175; (1999). 446, 349.

[85] Adams, G.S. et al. [E852 Collaboration] (2007). Confirmation of the $1^{-+}$meson exotics in the $\eta \pi^{0}$ System. Phys.Lett. B657, 27.

[86] Azizi, K., Barsbay, B. and Sundu, H. Mass and residue of $\Lambda(1405)$ as a hybrid baryon, arXiv:1705.10345 [hep-ph].

[87] Hall, J.M.M. et al. (2015). Lattice QCD evidence that the $\Lambda(1405)$ resonance is an antikaon-nucleon molecule, Phys. Rev. Lett. 114 (13) 132002.

[88] Dalitz, R. and Tuan, S. (1959). A possible resonant state in pion-hyperon scattering, Phys.Rev.Lett. 2, 425428.
[89] Dalitz, R. and Tuan, S. (1960). The phenomenological description of $K$ - nucleon reaction processes. Annals Phys. $10,307-351$

[90] Roca, L. and Oset, E. (2016). On the hidden charm pentaquarks in $\Lambda_{b} \rightarrow J / \psi K^{-} p$ decay. Eur.Phys.J. C76 (11) 591

[91] Bazzi, M. et al. (2012). Kaonic hydrogen X-ray measurement in SIDDHARTA, Nucl.Phys. A881, 88-97.

[92] Sumihama, M. et al. [LEPS Collaboration] (2006). The $\gamma p \rightarrow K^{+} \Lambda$ and $\gamma p \rightarrow K^{+} \Sigma^{0}$ reactions at forward angles with photon energies from $1.5 \mathrm{GeV}$ to $2.4 \mathrm{GeV}$. Phys.Rev. C73, 035214

[93] Moriya, K. et al. [CLAS Collaboration] (2013). Differential photoproduction cross sections of the $\Sigma^{0}(1385), \Lambda(1405)$ and $\Lambda(1520)$. Phys.Rev. C 88, 045201. Addendum: [Phys. Rev. C 88 (4) 049902 (2013)].

[94] Agakishiev, G. et al. [HADES Collaboration] (2013). Baryonic resonances close to the $\bar{K} N$ threshold: the case of

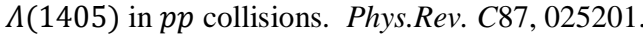

[95] Cheng, H.Y., Chua, C.K. and Liu, K.F. (2015). Revisiting scalar glueballs.Phys.Rev. D92 (9) 094006.

[96] Amsler, C. and Close, F.E. (1996). Is $f^{0}(1500)$ a scalar glueball? Phys. Rev. D53, 295.

[97] Azhinenko, I.V. et al. [CERN-USSR Collaboration] (1984). Inclusive $K^{*+}$ (890), $K^{*+}(1430)$ and $K^{*}(890)$ Production in $K^{+} p$ Interactions at $32 \mathrm{GeV} / c$. Z.Phys. $C 25$, 103.

[98] Abele, A. et al. [Crystal Barrel Collaboration] (1997). Study of the $\pi^{0} \pi^{0} \eta^{\prime}$ final state in $p p$ annihilation at rest. Phys.Lett. B404 179-186.

[99] Abele, A. et al. [Crystal Barrel Collaboration] (1998). $\bar{p} p$ annihilation at rest into $K_{L} K^{ \pm} \pi^{\mp}$. Phys. Rev. D 57, 3860 .

[100] Ablikim, M. et al. [BES Collaboration] (2006). Partial wave analyses of $J / \psi \rightarrow \gamma \pi^{+} \pi^{-}$and $\gamma \pi^{0} \pi^{0}$. Phys.Lett. B642, 441-448.

[101] Ablikim, M. et al. [BESIII Collaboration] (2013). Partial wave analysis of $J / \psi \rightarrow \gamma \eta \eta$. Phys.Rev. D87 (9) 092009, Erratum: Phys.Rev. D87 (2013) no.11, 119901.

[102] Rai, A.K. Rathaud, D.P. (2015). The mass spectra and decay properties of dimesonic states, using the Hellmann potential. Eur.Phys.J. C75 (9) 462.

[103] Adamo, A. et al. [OBELIX Collaboration] (1992). First physics results from OBELIX. Sov.J.Nucl.Phys. 55, 1732-1742, Yad.Fiz. 55, 3099-3121.

[104] Close, F.E. and Page, P.R. (1995). The production and decay of hybrid mesons by flux-tube breaking. Nucl. Phys. B 443, 23. 
[105] Adams, G.S. et al. [CLEO Collaboration] (2011). Amplitude analyses of the decays $\chi_{c 1} \rightarrow \eta \pi^{+} \pi^{-}$and $\chi_{c 1} \rightarrow \eta^{\prime} \pi^{+} \pi^{-}$. Phys.Rev. D84 112009.

[106] Ivanov, E. I. et al. [E852 Collaboration] (2001). Observation of exotic meson production in the reaction $\pi^{-} p \rightarrow \eta^{\prime} \pi^{-} p$ at $18 \mathrm{GeV} / c$. Phys.Rev.Lett. 86, 3977.

[107] Khokhlov, Y.A. et al. [VES Collaboration] (2000). Study of X(1600) $1^{-+}$hybrid. Nucl.Phys.A 663, 596.

[108] Amsler, C. et al. (2008). Review of particle physics. Phys.Lett. B 667, 1.

[109] Pinder, C.N. [CRYSTAL BARREL Collaboration] (1997). Analysis of $\bar{p} p \rightarrow \pi^{0} \pi^{0} \pi^{0} \eta$ for annihilation at rest in liquid and gaseous hydrogen targets. Nucl.Phys.Proc.Suppl. 56 (1) 154-159.

[110] Kalashnikova, Y.S. (1994). If the $\rho$ (1405) is the exotic $1^{-}\left(1^{-+}\right)$hybrid, then where are the nonexotic ones? Nuovo Cim. A107, 2421-2424.

[111] Klempt, E. and Zaitsev, A. (2007). Glueballs, hybrids, multiquarks. Experimental facts versus QCD inspired concepts. Phys. Rept. 454, 1.

[112] Albaladejo, M. and Oller, J.A. (2008). Identification of a scalar glueball. Phys. Rev. Lett. 101, 252002.

[113] Ghoul, H.A. et al. [GlueX Collaboration] (2017). Measurement of the beam asymmetry $\Sigma$ for $\pi^{0}$ and $\eta$ photoproduction on the proton at $E_{\gamma}=9 \mathrm{GeV}$. Phys.Rev. C95, 042201.

[114] Ablikim, M. et al. [BESIII Collaboration] (2013). Partial wave analysis of $J / \psi \rightarrow \gamma \eta \eta$. Phys.Rev. D87 (9) 092009. Erratum: Phys.Rev. D87 (2013) no.11, 119901.

[115] Bicudo, P. et al. (2007). The BES $f^{0}(1810)$ : A new glueball candidate. Eur.Phys.J. C52, 363-374.

[116] Ablikim, M. et al. [BES Collaboration] (2006). Observation of a near-threshold enhancement in the mass spectrum from the doubly OZI suppressed decay $J / \psi \rightarrow$ $\gamma \omega \varphi$. Phys. Rev. Lett. 96, 162002.

[117] Ablikim, M. et al. [BESIII Collaboration] (2015). Amplitude analysis of the $\pi^{0} \pi^{0}$ system produced in radiative $J / \psi$ decays. Phys.Rev. D 92, 052003.

[118] Amelin, D.V. et al. (2005). Investigation of hybrid states in the VES experiment at the Institute for High Energy Physics. Phys.Atom.Nucl. 68, 359-371, Yad.Fiz. 68 (2005) 388-400.

[119]Adolph, C. [COMPASS Collaboration] (2017). Resonance production and S-wave in $\pi^{-}+p \rightarrow$ $\pi^{-} \pi^{-} \pi^{+}+$precoil at $190 \mathrm{GeV} / c$. Phys.Rev. D95 (3) 032004 .

[120] Bing, C., Wei, K.W. and Zhang, A. (2013). $X(1870)$ and $\eta_{2}(1870)$ : Which can be assigned as a hybrid state? Adv. High Energy Phys. 2013, 217858.
[121] Anisovich, A.V. et al. [Crystal Barrel Collaboration] (2000). Three $I=0, J^{P C}=2^{-+}$mesons. Phys. Lett. B $477,19$.

[122] Barberis, D. et al. [WA102 Collaboration] (2000). A Study of the $\eta \pi^{+} \pi^{-}$channel produced in central $p p$ interactions at $450 \mathrm{GeV} / c$. Phys.Lett. B471, 435-439.

[123] Chen, Y.Q. and Li, X.Q. (2004). A comprehensive four-quark interpretation of $D_{S}(2317), D_{S}(2457)$ and $D_{s}$ (2632). Phys.Rev.Lett. 93232001.

[124] Cheng, H.Y. and Hou, W.S. (2003). B decays as spectroscope for charmed four quark states, Phys. Lett. B566 193-200.

[125] Browder, T.E., Pakvasa, S. and Petrov, A.A. Comment on the new $D_{s}^{(*)+} \pi^{0}$ resonances, Phys. Lett.B578 (2004) 365-368.

[126] B. Aubert et al. [BaBar collaboration] (2003). Observation of a narrow meson decaying to $D_{S}^{+} \pi^{0}$ at a mass of $2.32 \mathrm{GeV} / \mathrm{c}^{2}$. Phys. Rev. Lett. 90, 242001.

[127] Krokovny, P. et al., [Belle collaboration] (2003). Observation of the $D_{s J}(2317)$ and $D_{S J}(2457)$ in B decays. Phys. Rev. Lett. 91, 262002.

[128] Besson, D. et al. [CLEO collaboration] (2003). Observation of a narrow resonance of mass $2.46 \mathrm{GeV} / \mathrm{c}^{2}$ decaying to $D_{S}^{*+} \pi^{0}$ and confirmation of the $D_{S J}^{*}(2317)$ state, Phys. Rev. D68, 032002 [Erratum ibid. D 75 (2007) 119908].

[129] Vaandering, E.W. (2004). Charmed hadron spectroscopy from FOCUS, in: QCD and high energy hadronic interactions. Proceedings, 39th Rencontres de Moriond, La Thuile, Italy, March 28-April 2, pp. 127-132. arXiv:hep-ex/0406044.

[130] Yang, G. and Ping, J. The structure of pentaquarks $\Omega_{c}^{0}$ in the chiral quark model. Mar 26, 2017. 8 pp. arXiv:1703.08845 [hep-ph].

[131] Huang, H., Ping, J. and Wang, F. Investigating the excited $\Omega_{c}^{0}$ states through $\Xi_{c} K$ and $\Xi_{c}^{\prime} c K$ decay channels. Apr 5, 2017. 6 pp. arXiv:1704.01421 [hep-ph].

[132] Aaij, R.et al. [LHCb Collaboration] (2017). Observation of five new narrow $\Omega_{c}^{0}$ states decaying to $\Xi_{c}^{+} K$. Phys.Rev.Lett. 118 (18) 182001.

[133] Govaerts, J., Viron, F.D., Gusbin, D. and Weyers, J. (1984). QCD sum rules and hybrid mesons. Nucl.Phys. B248, 1-18.

[134] Bhardwaj, V. et al. [Belle Collaboration] (2013). Evidence of a new narrow resonance decaying to $\chi_{1 c} \gamma$ in $B \rightarrow \chi_{c 1} \gamma$ K. Phys.Rev.Lett. 111 (3) 032001.

[135] Ablikim, M. et al. [BESIII Collaboration] (2015). Observation of the $\psi\left(1^{3} D^{2}\right)$ state in $e^{+} e^{-} \rightarrow \pi^{+} \pi^{-} \gamma \chi_{1 c}$ at BESIII. Phys.Rev.Lett. 115 (1) 011803.

[136] Antoniazzi, L. et al. [E705 Collaboration] (1994). Search for hidden charm resonance states decaying into $J / \psi$ or $\psi$ ' plus pions. Phys.Rev. D50, 4258-4264. 
[137] Li, B.A. (2005). Is $X(3872)$ a possible candidate of hybrid meson. Phys.Lett. B605, 306-310.

[138] Voloshin, M.B. (2004). Heavy quark spin selection rule and the properties of the $X(3872)$. Phys.Lett. B604, 69-73.

[139] Burns, T.J., Piccinini, F., Polosa, A.D. and Sabelli, C. (2010). The $2^{-+}$assignment for the $\mathrm{X}(3872)$. Phys.Rev. D82, 074003.

[140] Maiani, L., Piccinini, F., Polosa, A. D. and Riquer, V. (2005). Diquark-antidiquarks with hidden or open charm and the nature of X(3872). Phys. Rev. D71, 014028 .

[141] Aubert, B. [BaBar Collaboration] (2004). Observation of the decay $B \rightarrow J / \psi \eta K$ and search for $X(3872) \rightarrow J / \psi \eta$. Phys.Rev.Lett. 93, 041801.

[142] Abazov, V. M. et al. [D0 Collaboration] Observation and properties of the $X(3872)$ decaying to $J / \psi \pi^{+} \pi^{-}$ inpp collisions at $\sqrt{s}=1.96 \mathrm{TeV}$, Phys. Rev. Lett. 93 (2004) 162002

[143] Ikeda, Y. [HAL QCD Collaboration] (2017). The Tetraquark candidate $Z_{c}(3900)$ from Dynamical Lattice QCD Simulations. arXiv:1706.07300 [hep-lat].

[144] Ablikim, M. et al. [BESIII Collaboration] (2015). Observation of $Z_{c}(3900)^{0}$ in $e^{+} e^{-} \rightarrow \pi^{0} \pi^{0} J / \psi$. Phys.Rev.Lett. 115 (11) 112003

[145] Dobbs, S. et al. [CLEO Collaboration] (2007). Measurement of absolute hadronic branching fractions of $D$ mesons and $e^{+} e^{-} \rightarrow$ DD cross-sections at the $\psi(3770)$. Phys.Rev. D76, 112001.

[146] Hogaasen,H., Kou, E., Richard, J.M. and Sorba, P. (2014). Isovector and hidden-beauty partners of the X(3872). Phys. Lett. B 732, 97.

[147] Karliner, M., Rosner, J.L. (2015). New exotic meson and baryon resonances from doubly-heavy hadronic molecules, Phys. Rev. Lett. 115 (12) 122001.

[148] Uehara, S. et al., [Belle Collaboration] (2010). Observation of a charmonium-like enhancement in the $\gamma \gamma \rightarrow \omega J / \psi$ process. Phys. Rev. Lett. 104, 092001.

[149] Lees, J.P. et al. [BaBar Collaboration] (2012). Study of $X(3915) \rightarrow J / \psi \omega$ in two-photon collisions, Phys. Rev. D86, 072002

[150] Liao, X. and Manke, T. (2002). Excited charmonium spectrum from anisotropic lattices, CU-TP-1063, arXiv:hep-lat/0210030v2.

[151] Close, F.E., and Godfrey, S.. (2003). Charmonium hybrid production in exclusive $B$ meson decays. Phys.Lett. B574, 210-216.

[152] Branz, T., Gutsche, T. and Lyubovitskij, V.E. (2009). Hadronic molecule structure of the $Y(3940)$ and Y(4140). Phys.Rev. D80, 054019.
[153]Pakhlov, P. [Belle Collaboration] (2008). Production of new charmoniumlike states in $e^{+} e^{-} \rightarrow J / \psi D^{(*)} \bar{D}^{(*)}$ at $\sqrt{s} \approx 10.6 \mathrm{GeV}$. Phys.Rev.Lett. 100, 202001

[154] Aubert, B. et al., [BaBar Collaboration] (2008). Observation of $Y(3940) \rightarrow J / \psi \omega$ in $B \rightarrow J / \psi \omega K$ at BABAR. Phys. Rev. Lett. 101, 082001.

[155] Wang, Z.G. (2016). Tetraquark state candidates: $Y(4260), Y(4360), Y(4660) \quad$ and $\quad Z_{c}(4020 /$ 4025) Eur.Phys.J. C76 (7) 387.

[156] Yuan, C.Z. [BESIII Collaboration] (2015). Study of the $X Y Z$ states at the BESIII. Front.Phys. (Beijing) 10 (6) 101401.

[157] Ablikim, M. et al. [BESIII Collaboration] (2014). Observation of $e^{+} e^{-} \rightarrow \pi^{0} \pi^{0} h_{c}$ and a neutral charmoniumlike structure $Z_{c}(4020)^{0}$. Phys. Rev. Lett. 113,212002

[158] Oncala, R. and Soto, J. (2017). Heavy quarkonium hybrids: Spectrum, decay and mixing. Phys.Rev. D96 (1) 014004

[159] Chen, X. et al. (2016). Calculation of mass of $Y(4140)$ by introducing mixed molecule state in quark model. Nucl.Phys. B909, 243-259.

[160] Dăg, H. and A. Türkan. (2017). Investigating the structure of $X(4140)$ in QCD. EPJ Web Conf. 137, 06007.

[161] Agaev, S.S. Azizi, K. and Sundu, H. (2017). Exploring the resonances $X(4140)$ and $X(4274)$ through their decay channels. Phys.Rev. D95, (11) 114003.

[162] Wang, Z.G. (2016). Reanalysis of $X(4140)$ as axialvector tetraquark state with QCD sum rules. Eur.Phys.J. C76 (12) 657.

[163] Aaltonen, T. et al., [CDF Collaboration] (2009). Evidence for a narrow near-threshold structure in the $J / \psi \phi$ mass spectrum in $B^{+} \rightarrow J / \psi \phi K^{+}$decays, Phys. Rev. Lett. $102,242002$.

[164] Chatrchyan, S. et al., [CMS Collaboration] (2014). Observation of a peaking structure in the $J / \psi \phi$ mass spectrum from $B^{ \pm} \rightarrow J / \psi \phi K^{ \pm}$decays, Phys. Lett. B734, 261-281

[165] Abazov, V.M. [D0 Collaboration] (2014). Search for the $X(4140)$ state in $B^{+} \rightarrow J / \psi \phi K^{+}$decays with the D0 Detector. Phys.Rev. D89 (1) 012004.

[166] Prencipe, E. [B AB AR Collaboration] (2015). Search for exotics in the rare decay $B \rightarrow J / \psi K K K$ at BABAR. EPJ Web Conf. 95, 05012.

[167] Chen, Y., Chiu, W.F., Gong, M., Gui, L.C. and Liu, Z. (2016). Exotic vector charmonium and its leptonic decay width. Chin. Phys. C40 (8) 081002.

[168] Wang, Z.G. (2017). Analysis of the $Y(4220)$ and $Y(4390)$ as molecular states with QCD sum rules. Chin.Phys. C41 (8) 083103. 
[169] Feng, X.N., Gao, X.Y. and Shen, C.P. (2015). Combined fit to BESIII data on $e^{+} e^{-} \rightarrow h_{c} \pi^{+} \pi^{-}$and $\chi_{c 0} \omega$. Int.J.Mod.Phys. A30 (24) 1550142.

[170] Wang, X.L. et al. [Belle Collaboration] (2015). Measurement of $e^{+} e^{-} \rightarrow \pi^{+} \pi^{-} \psi(2 S)$ via initial state radiation at Belle, Phys. Rev. D 91, 112007.

[171] Ablikim, M. et al. [BESIII Collaboration] (2015). Study of $e^{+} e^{-} \rightarrow \omega \chi_{c J}$ at center-of-mass energies from 4.21 to $4.42 \mathrm{GeV}$, Phys. Rev. Lett. 114, 092003.

[172] Zhu, S.L. (2008). New hadron states. Int.J.Mod.Phys. E17, 283-322.

[173] Qiao, C.F. (2006). One explanation for the exotic state $Y(4260)$. Phys.Lett. B639, 263-265.

[174] Lees, J.P. [BaBar Collaboration] (2012). Study of the reaction $e^{+} e^{-} \rightarrow J / \psi \pi^{+} \pi^{-}$via initial-state radiation at BaBar. Phys.Rev. D86, 051102.

[175] He, Q. [CLEO Collaboration] (2006). Confirmation of the $Y(4260)$ resonance production in ISR. Phys.Rev. D74, 091104 .

[176] Wang, M.Z. et al. [BELLE Collaboration] (2005). Study of the baryon-antibaryon low-mass enhancements in charmless three-body baryonic B decays. Phys. Lett. B617, 141 .

[177] Lees, J.P. et al. [BaBar Collaboration] (2014). Study of the reaction $e^{+} e^{-} \rightarrow \psi(2 S) \pi^{-} \pi^{-}$via initialstate radiation at BaBar, Phys. Rev. D89 (11) 111103.

[178] Wang X.L. et al., [Belle Collaboration] (2007). Observation of two resonant structures in $e^{+} e^{-} \rightarrow$ $\pi^{+} \pi^{-} \psi(2 S)$ via initial state radiation at Belle, Phys. Rev. Lett. 99, 142002.

[179] He, J. $D \Sigma_{c}^{*}$ and $D^{*} \Sigma_{c}$ interactions and the LHCb hidden-charmed pentaquarks. Phys.Lett. B753 (2016) 547 551.

[180] Azizi, K. Sarac, Y. and Sundu, H. Hidden bottom pentaquark states with spin $3 / 2$ and $5 / 2$. arXiv:1707.01248v1 [hep-ph].

[181] Deng, C., Ping, J., Huang, H. and Wang, F. (2015). Systematic study of $Z_{c}^{+}$family from a multiquark color flux-tube model, Phys. Rev. D92 (3) 034027.

[182] Chilikin, K. [Belle Collaboration] (2013). Experimental constraints on the spin and parity of the Z(4430) ${ }^{+}$. Phys.Rev. D88, (7) 074026.

[183] Wang, Z.G. (2017). Scalar tetraquark state candidates: $X(3915), X(4500)$ and $X(4700)$. Eur.Phys.J. C77 (2) 78 .

[184] Aaij, R. [LHCb Collaboration] (2017). Amplitude analysis of $B^{+} \rightarrow J / \psi \phi K^{+}$decays. Phys.Rev. D95, (1) 012002 .

[185] Oncala, R. and Soto, J. (2017). Heavy hybrids: Decay to and mixing with heavy quarkonium. EPJ Web Conf. 137, 06025.
[186] Wang, Z.G. and Zhang, X.H. (2010). Analysis of $Y(4660)$ and related bound states with QCD sum rules. Commun. Theor. Phys. 54, 323.

[187] Aubert, B. et al. [BaBar Collaboration] (2007). Evidence of a broad structure at an invariant mass of $4.32 \mathrm{GeV} / \mathrm{c}^{2}$ in the reaction $e^{+} e^{-} \rightarrow \pi^{+} \pi^{-} \psi(2 S)$ measured at BaBar. Phys. Rev. Lett. 98, 212001.

[188] Albaladejo, M., Nieves, J., Oset, E., Sun, Z.F. and Liu, X. (2016). $X(5568)$ be described as a $B_{S} \pi, B \bar{K}$ resonant state? Phys.Lett. B757, 515-519.

[189] Agaev, S.S., Azizi, K. and Sundu, H. (2016). Mass and decay constant of the newly observed exotic X(5568) state, Phys. Rev. D93 (7) 074024.

[190] Zhang, J.R., Zou, J.L. and Wu, J.Y. $0^{+}$tetraquark states from improved QCD sum rules: Delving into $X(5568)$.May 10, 2017. 14 pp. e-Print: arXiv:1705.03741 [hep-ph].

[191] Krokovny, P. [Belle Collaboration] (2013). First observation of the $Z_{b}(10610)$ in a Dalitz analysis of $\Upsilon(10860) \rightarrow \Upsilon(n S) \pi^{0} \pi^{0}$. Phys.Rev. D88, (5) 052016.

[192] Wang, Z.G. and Huang, T. (2014). The $Z_{b}(10610)$ and $Z_{b}(10650)$ as axial-vector tetraquark states in the QCD sum rules. Nucl.Phys. A930, 63-85.

[193] Guo, F.K., Hidalgo-Duque, C., Nieves, J., Ozpineci, A. and Valderrama, M.P. (2015). Hidden charm and bottom molecular states. Hyperfine Interact. 234 (1-3) 125-132.

[194] Bondar et al. [Belle Collaboration] (2012). Observation of two charged bottomonium-like resonances in $Y(5 S)$ decays. Phys. Rev. Lett. 108, 122001. 